\title{
Decay constants of $B$ and $D$ mesons from improved relativistic lattice QCD with two flavors of sea quarks
}

A. Ali Khan, ${ }^{1}$ S. Aoki, ${ }^{2}$ R. Burkhalter, ${ }^{1,2}$ S. Ejiri, ${ }^{1}$ M. Fukugita,${ }^{3}$ S. Hashimoto, ${ }^{4}$ N. Ishizuka, ${ }^{1,2}$ Y. Iwasaki, ${ }^{1,2}$ K. Kanaya, ${ }^{1,2}$ T. Kaneko, ${ }^{4}$ Y. Kuramashi, ${ }^{4}$ T. Manke, ${ }^{5}$ K. Nagai, ${ }^{1}$ M. Okawa, ${ }^{4}$ H. P. Shanahan, ${ }^{1,6}$ A. Ukawa, ${ }^{1,2}$ and T. Yoshie ${ }^{1,2}$

(CP-PACS Collaboration)

${ }^{1}$ Center for Computational Physics, University of Tsukuba, Tsukuba, Ibaraki 305-8577, Japan

${ }^{2}$ Institute of Physics, University of Tsukuba, Tsukuba, Ibaraki 305-8571, Japan

${ }^{3}$ Institute for Cosmic Ray Research, University of Tokyo, Tanashi, Tokyo 188-8502, Japan

${ }^{4}$ High Energy Accelerator Research Organization (KEK), Tsukuba, Ibaraki 305-0801, Japan

${ }^{5}$ Department of Physics, Columbia University, 538 West 120th Street, New York, New York 10027

${ }^{6}$ Department of Biochemistry and Molecular Biology, University College London, Darwin Building, Gower Street, London, WC1E 6BT, United Kingdom

(Received 9 October 2000; published 3 July 2001)

\begin{abstract}
We present a calculation of the $B$ and $D$ meson decay constants in lattice QCD with two $\left(N_{f}=2\right)$ flavors of light dynamical quarks, using an $O(a)$-improved Wilson action for both light and heavy quarks and a renormalization-group improved gauge action. Simulations are made at three values of lattice spacing $a$ $=0.22,0.16,0.11 \mathrm{fm}$ and four values of sea quark mass in the range $m_{P S} / m_{V} \approx 0.8-0.6$. Our provisional estimate for the continuum values of the decay constants are $f_{B d}=208(10)(29) \mathrm{MeV}, f_{B s}$ $=250(10)(35)\left(\begin{array}{c}+8 \\ -0\end{array}\right) \mathrm{MeV}, f_{D d}=225(14)(40) \mathrm{MeV}, f_{D s}=267(13)(48)\left({ }_{-0}^{+10}\right) \mathrm{MeV}$ for $N_{f}=2$ where the statistical and systematic errors are separately listed, and the third error for $f_{B s}$ and $f_{D s}$ shows the uncertainty of the determination of the strange quark mass. We also carry out a set of quenched simulations using the same action to make a direct examination of sea quark effects. Taking the ratio of results for $N_{f}=2$ and $N_{f}=0$, we obtain $f_{B d}^{N_{f}=2} / f_{B d}^{N_{f}=0}=1.11(6), f_{B s}^{N_{f}=2} / f_{B s}^{N_{f}=0}=1.14(5), f_{D d}^{N_{f}=2} / f_{D d}^{N_{f}=0}=1.03(6), f_{D s}^{N_{f}=2} / f_{D s}^{N_{f}=0}=1.07(5)$. They show a 10$15 \%$ increase in the $N_{f}=2$ results over those of $N_{f}=0$ for the $B$ meson decay constants, while evidence for such a trend is statistically less clear for the $D$ meson decay constants.
\end{abstract}

DOI: 10.1103/PhysRevD.64.034505

\section{INTRODUCTION}

The accurate determination of the Cabibbo-KobayashiMaskawa (CKM) matrix elements is one of the most important tasks of flavor physics. The standard model prediction of the unitarity of the matrix still has to be tested, especially for the unitarity relation involving the most off-diagonal elements, which contain the source of the $C P$ violation in the standard model.

Two of the matrix elements $\left|V_{t d}\right|$ and $\left|V_{t s}\right|$ can be extracted from the experimental data of the oscillation frequency $\Delta m_{q}$ of $B_{q}-\bar{B}_{q}$ systems ( $q$ denotes either $d$ or $s$ quark) through the relation [1]

$$
\Delta m_{q}=\frac{G_{F}^{2}}{6 \pi^{2}} M_{W}^{2} S_{0}\left(x_{t}\right) \eta_{B} M_{B_{q}} f_{B_{q}}^{2} \hat{B}_{B_{q}}\left|V_{t q} V_{t b}^{*}\right|^{2},
$$

where the factors other than $f_{B_{q}}^{2} \hat{B}_{B_{q}}$ are known either experimentally or through perturbative calculations in QCD. The nonperturbative coefficients $f_{B_{q}}$ and $\hat{B}_{B_{q}}$ are defined as

$$
\left\langle 0\left|\bar{b} \gamma_{\mu} \gamma_{5} q\right| B_{q}(p)\right\rangle=i f_{B_{q}} p_{\mu}
$$

and

where $R_{B}(\mu)$ denotes a renormalization group factor to eliminate the variation due to the scale $\mu$ where the fourquark operator $\bar{b} \gamma_{\mu}\left(1-\gamma_{5}\right) q \bar{b} \gamma_{\mu}\left(1-\gamma_{5}\right) q$ is defined. In this paper we shall focus on the decay constants $f_{B_{q}}$, leaving the bag parameter $\hat{B}_{B_{q}}$ for future studies.

Experimentally, the Cabibbo-allowed leptonic decay $D_{s}$ $\rightarrow \tau \bar{\nu}_{\tau}$ has been measured and the recent values for $f_{D_{s}}$ are

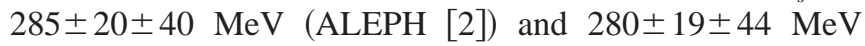
(CLEO [3]). On the other hand, a measurement of the decay constant $f_{B}$ is difficult, since $B^{+} \rightarrow l^{+} \bar{\nu}_{l}$ is Cabibbo suppressed in the standard model. Hence $f_{B_{q}}$ has to be provided from theory, while $f_{D_{q}}$ can be used to check the calculational method.

The calculation of these decay constants have been carried out extensively in the quenched approximation in lattice QCD, where vacuum polarization effects are neglected in order to reduce the computational requirements. A recent summary of these attempts is given in Refs. [4,5]. Although the approximation provides a useful first step in a lattice QCD determination of the decay constants, the size of the resulting systematic error is not clear. In the quark potential model, the decay constant is proportional to the wave func- 
tion at the origin squared and the potential at such short distance scales can be expressed in terms of the running coupling constant. Therefore, one can demonstrate, on a heuristic level, that the decay constant is affected by the number of flavors $N_{f}$, where $N_{f}=0$ corresponds to the quenched approximation. Additionally, a recent study of the light hadron spectrum in the quenched approximation indicates a deviation of about $10 \%$ from experiment [6]. For the decay constants, quenched chiral perturbation theory suggests $[7,8]$ that the deviation introduced by the approximation may be significant.

The elimination of this approximation is numerically highly intensive and has become realistic only recently. The MILC Collaboration [9] and Collins et al. [10] have performed first calculations of the decay constants on the lattice with two degenerate sea quark flavors, and found an indication that $f_{B_{q}}$ is considerably larger in the presence of sea quarks. In these studies the discretization of the sea quarks is defined using the staggered fermion action, which is different from that used for the light valence quark [Wilson fermion in Ref. [9] and the $O(a)$-improved (clover) fermion in Ref. [10]]. It could introduce an additional source of systematic error in the results. In fact, a rather different $a$ (the lattice spacing), dependence in $f_{B}$ is observed in Ref. [9] between quenched and unquenched calculations, even though the formulations for valence heavy and light quarks are the same.

In our work we apply a consistent formulation where for both sea and light valence quarks we use the same action, and study the $a$ dependence by performing three sets of twoflavor calculations at $a \simeq 0.22,0.16$, and $0.11 \mathrm{fm}$. For comparison, we carry out quenched calculations at ten different values of $a$ covering the range studied in the two flavor calculations. We employ the $O(a)$-improved quark action [11] for both sea and valence light quarks. The same action is used for the heavy quark, applying the nonrelativistic reinterpretation of Ref. [12]. The gauge field is described by a renormalization group improved action [13], which reduces the descretization error on the coarse lattices on which our calculations are made.

The rest of this paper is outlined as follows. In Sec. II we discuss the lattice actions and the formulation to treat heavy quarks. The computational details involved in the calculation are described in Sec. III, and our analysis procedures in Sec. IV. We present the results in Sec. V where we discuss in particular how we estimate the values in the continuum limit and their errors, and make a comparison between the $N_{f}$ $=0$ and $N_{f}=2$ results. Our conclusions are summarized in Sec. VI.

\section{LATTICE ACTIONS}

\section{A. Light sector}

The renormalization group (RG) improved gauge action we employ takes the form [13]

$$
S_{g}^{\mathbf{R}}=\frac{\beta}{6}\left(c_{0} \sum W_{1 \times 1}+c_{1} \sum W_{1 \times 2}\right),
$$

where $W_{1 \times 1}$ and $W_{1 \times 2}$ are the Wilson loops of size $1 \times 1$ and $1 \times 2$, respectively, and the sums run over all possible sites and orientations. The parameter $\beta$ is related to the bare gauge coupling $g_{0}^{2}$ through $\beta=6 / g_{0}^{2}$. The coefficients $c_{0}$ and $c_{1}$ are defined as

$$
\begin{aligned}
& c_{0}=3.648, \\
& c_{1}=\frac{1}{8}\left(1-c_{0}\right)=-0.331,
\end{aligned}
$$

which are chosen so as to approximate the renormalization group trajectory in two dimensional operator space.

For quarks we employ the $O(a)$-improved (clover) action [11] defined by

$$
S_{q}^{\mathbf{C}}=\sum_{x, y} \bar{\psi}_{x}\left[D_{x y}^{\mathbf{W}}-c_{S W} K \sum_{\mu<\nu} \sigma_{\mu \nu} F_{\mu \nu}\right] \psi_{y},
$$

where $D_{x y}^{\mathrm{W}}$ is the standard Wilson formulation of the Dirac fermion matrix

$$
\begin{aligned}
D_{x y}^{\mathbf{W}}= & \delta_{x y}-K \sum_{\mu}\left\{\left(1-\gamma_{\mu}\right) U_{x, \mu} \delta_{x+\hat{\mu}, y}\right. \\
& \left.+\left(1+\gamma_{\mu}\right) U_{x, \mu}^{\dagger} \delta_{x, y+\hat{\mu}}\right\}
\end{aligned}
$$

and the matrix $F_{\mu \nu}$ is the simplest definition of the field strength,

$$
F_{\mu \nu}=\frac{1}{8 i}\left(f_{\mu \nu}-f_{\mu \nu}^{\dagger}\right),
$$

where $f_{\mu \nu}$ is the standard clover-shaped definition of the gauge field strength. The leading discretization error in the Wilson fermion action $\left(c_{S W}=0\right)$ is removed by appropriately tuning the parameter $c_{S W}$. We apply a mean field approximation $c_{S W}=P^{-3 / 4}$, where $P=\left\langle W_{1 \times 1}\right\rangle$. To avoid a tuning of $c_{S W}$ depending on the hopping parameter $K$, a perturbative expansion at one-loop $P=1-0.1402 g_{0}^{2}$ is used to evaluate $P$, since we find that the one-loop estimate approximates the observed value of $P$ very well for our range of parameters, the difference being at worst $8 \%$ [14]. Furthermore, there is also good agreement between the above definition of $c_{S W}$ and the one-loop value computed in Ref. [15]. With this choice, the leading contributions among remaining discretization errors are $O\left(\alpha_{s} a\right)$ and $O\left(a^{2}\right)$ for light quarks.

The efficacy of this choice of actions over the standard action has been demonstrated in Ref. [16] by examining the rotational invariance of the static potential and the scaling behavior of the light hadron spectrum. In using the clover fermion action, we also note that care must be taken in defining currents, which will be discussed below.

\section{B. Heavy quarks}

It seems implausible to examine hadrons containing heavy quarks with mass $m_{Q} a>1$ on a lattice with the spacing $a$, as one expects the discretization effects to become uncontrollably large for such large masses. However, this is 
not necessarily true for heavy-light mesons. The spatial momentum of the light degrees of freedom in the heavy-light system is controlled by the QCD scale $\Lambda_{\mathrm{QCD}}$ rather than the much larger heavy quark mass scale. In the limit of infinite $m_{Q}$, the heavy quark mass decouples from the dynamics of the system, and the heavy quark effective theory (HQET) [17] becomes a good approximation. At a finite $m_{Q}$, the correction may be incorporated as an expansion in $1 / m_{Q}$, which is a basis of the nonrelativistic QCD (NRQCD).

On the lattice, it is straightforward to formulate the static [18] and NRQCD [19,20] actions, and a number of (quenched) calculations of $f_{B}$ have been performed using them. Another formulation to realize the idea of HQET on the lattice [12] is also useful, as it uses the same relativistic form of the quark action as that for light quarks except that the bare heavy quark mass $m_{0}$ may be taken to be arbitrarily large.

For the heavy-light system, where the typical spatial momentum of the heavy quark is small compared to the inverse lattice spacing, one can construct an effective Hamiltonian starting from a relativistic action

$$
\hat{H} \approx \hat{\bar{\Psi}}\left[M_{1}+\gamma_{0} A_{0}-\frac{\mathbf{D}^{2}}{2 M_{2}}-\frac{i \mathbf{\Sigma} \cdot \mathbf{B}}{2 M_{B}}-\gamma_{0} \frac{[\gamma \cdot \mathbf{D}, \gamma \cdot \mathbf{E}]}{8 M_{E}^{2}}\right] \hat{\Psi},
$$

where $\mathbf{D}$ is the covariant derivative, $\boldsymbol{\Sigma}$ the Pauli spin matrices, and $\mathbf{B}$ and $\mathbf{E}$ are the chromomagentic and chromoelectric fields, respectively, and an expansion in small spatial momentum or equivalently in $a \mathbf{D}$ on the lattice is performed. This Hamiltonian is equivalent to the standard nonrelativistic Hamiltonian if the "mass" parameters $M_{1}, M_{2}, M_{B}$, and $M_{E}$ are equal to each other. Those are, however, different functions of $a m_{0}$ and not necessarily equal to each other, unless the parameters in the initial relativistic action are appropriately tuned. The strategy suggested in Ref. [12] is, therefore, to take the action as an effective theory to generate the dynamics described by Eq. (10). The appropriate mass parameter in the nonrelativistic effective theory is the " $\mathrm{ki}$ netic" mass $M_{2}$, while the "pole" mass $M_{1}$ does not affect the dynamics of heavy quark and plays merely a role of energy shift in this formalism. To obtain a correct action at order $1 / M$, the mass parameter which characterizes the spinmagnetic interaction $M_{B}$ must be equal to $M_{2}$, which is satisfied for the $O(a)$-improved (clover) action up to perturbative corrections. On the other hand, there is no tunable parameter in the clover action to make $M_{E}$ equal to $M_{2}$ and $M_{B}$, so that the contributions of $O\left(1 / M^{2}\right)$ and higher are not correctly described by the clover action.

At tree level, the kinetic mass $M_{2}$ of the heavy quark is given by [12]

$$
a M_{2}=\left(\frac{2}{a m_{0}\left(2+a m_{0}\right)}+\frac{1}{1+a m_{0}}\right)^{-1},
$$

where the bare mass $a m_{0}$ is defined as $a m_{0}=\frac{1}{2}(1 / K$ $\left.-1 / K_{c}\right)$. The one-loop relation is also known [21-23] for the standard plaquette gauge action but not for our choice of the action. Hence we employ the tadpole improvement [24] of the above relation, which is obtained by simply replacing $a m_{0}$ with $8 K_{c} a m_{0}$.

The heavy-light meson mass $a M_{\mathrm{HQET}}$ defined in the HQET is then obtained as $[25,26]$

$$
a M_{\mathrm{HQET}}^{M}=a M_{\mathrm{pole}}^{M}+\left(a M_{2}^{Q}-a M_{1}^{Q}\right),
$$

from the pole mass $a M_{\text {pole }}$ extracted from the exponential fall off of the heavy-light propagator. The superscript $M$ or $Q$ in Eq. (12) distinguishes the mass of the heavy-light meson $(M)$ from the heavy quark mass $(Q)$. The parameters $a M_{1}^{Q}$ and $a M_{2}^{Q}$ are the tree-level defined pole and kinetic masses of the heavy quark.

An alternative way to obtain the heavy-light meson mass is to measure its energy-momentum dispersion relation and fit with the form $E(\mathbf{p})=M_{\text {pole }}+\mathbf{p}^{2} /\left(2 M_{\text {kin }}\right)+O\left(\mathbf{p}^{4}\right)$ to extract the "kinetic" mass $M_{\text {kin }}$ (as employed in Ref. [27]). Unfortunately, for the lattices which were used to quote our final results the statistical ensemble was not large enough to obtain an accurate measurement of $M_{\text {kin }}$. For this reason, this choice of the kinetic mass will not be further discussed here.

The axial current to be measured should also be modified to obtain the results correct at $O(1 / M)$ according to

$$
h \rightarrow\left(1-a d_{1} \gamma \cdot \mathbf{D}\right) h,
$$

where $h$ is the heavy quark field and equivalently for $\bar{h}$, and the parameter $d_{1}$ is a function of $a m_{0}$. At the tree level, it is given by [12]

$$
a d_{1}=\frac{1+a m_{0}}{a m_{0}\left(2+a m_{0}\right)}-\frac{1}{2 a M_{2}},
$$

and the axial vector current for heavy-light mesons, correct to $O(1 / M)$, takes the form

$$
A_{\mu}(x)=\bar{l}(x) \gamma_{5} \gamma_{\mu} h(x)-a d_{1} \bar{l}(x) \gamma_{5} \gamma_{\mu} \gamma \cdot \Delta h(x),
$$

where $l$ is the light quark field. The tadpole improvement of $d_{1}$ may be applied again with the replacement $a m_{0}$ $\rightarrow 8 K_{c} a m_{0}$.

The following point should also be noted. The action being used is still a relativistic action and as the lattice spacing becomes smaller, it is expected that theory should smoothly cross over to a fully relativistic theory. That means the mass parameters $M$ 's become identical as $a m_{0}$ decreases. The lattice spacing dependence of physical quantities, such as $f_{B}$ and $f_{D}$, is, however, highly nontrivial unless $m_{0}$ is much smaller than $1 / a$, and the continuum extrapolation in such a situation would not be justified with any simple ansatz, e.g., linear or quadratic in $a$. The formulation is, therefore, treated as an effective theory (such as NRQCD), and the discretization error should be reasonably small at fixed $a$ in order to obtain reliable results.

Despite the caveat of the preceding paragraph, this approach has been successfully implemented in the quenched approximation in Refs. $[26,27]$ using the plaquette gauge action. Since we use a gauge action which has been unused in 
TABLE I. Simulation parameters for $N_{f}=2$ lattices used in the heavy quark calculation. For the number of trajectories those in parentheses show the full ensemble generated.

\begin{tabular}{ccccccc}
\hline \hline$\beta$ & $c_{S W}$ & $K_{\text {sea }}$ & $m_{P S} / m_{V}$ & No. traj. & $K_{s}(K)^{-1}$ & $K_{s}(\phi)^{-1}$ \\
\hline 1.8 & 1.60 & 0.1409 & $0.807(1)$ & $5680(6250)$ & $6.929(3)$ & $7.037(6)$ \\
& & 0.1430 & $0.753(1)$ & $5200(5200)$ & $6.945(4)$ & $7.045(8)$ \\
& & 0.1445 & $0.694(2)$ & $6530(7000)$ & $6.956(3)$ & $7.044(7)$ \\
& & 0.1464 & $0.547(4)$ & $4070(5250)$ & $6.969(4)$ & $7.028(8)$ \\
1.95 & 1.53 & 0.1375 & $0.804(1)$ & $6810(7000)$ & $7.144(2)$ & $7.190(3)$ \\
& & 0.1390 & $0.752(1)$ & $5000(7000)$ & $7.154(2)$ & $7.196(3)$ \\
& & 0.1400 & $0.690(1)$ & $6800(7000)$ & $7.164(2)$ & $7.202(3)$ \\
& & 0.1410 & $0.582(3)$ & $4870(7000)$ & $7.166(2)$ & $7.195(4)$ \\
2.1 & 1.47 & 0.1357 & $0.810(2)$ & $1990(4000)$ & $7.283(3)$ & $7.306(6)$ \\
& & 0.1367 & $0.757(3)$ & $2000(4000)$ & $7.282(2)$ & $7.298(4)$ \\
& 0.1374 & $0.693(3)$ & $1910(4000)$ & $7.285(2)$ & $7.299(4)$ \\
& 0.1382 & $0.571(6)$ & $1945(4000)$ & $7.285(3)$ & $7.299(5)$ \\
\hline
\end{tabular}

the previous heavy quark calculations it is important for us that we repeat the calculation in the quenched approximation in order to see if the quenched results obtained with the "standard" plaquette gauge action are reproduced.

\section{COMPUTATIONAL DETAILS}

\section{A. Gauge fields}

Gauge configurations were generated for $N_{f}=0$ and $N_{f}$ $=2$ using the renormalization group $(\mathrm{RG})$ improved gauge action and the $O(a)$-improved Wilson quark action as discussed in Sec. II A. Technical details on the configuration generation for $N_{f}=2$, carried out with the hybrid Monte Carlo algorithm, are described in our dynamical QCD calculations papers $[28,29,14]$.

In the $N_{f}=2$ calculations, we performed three sets of calculations at bare gauge couplings $\beta=1.8,1.95$, and 2.1, which correspond to the lattice spacing $a \sim 0.22,0.16$, and $0.11 \mathrm{fm}$, respectively. The lattice size is $12^{3} \times 24(\beta=1.8)$, $16^{3} \times 32(1.95)$, and $24^{3} \times 48(2.1)$, with which the physical volume is approximately $(2.5 \mathrm{fm})^{3}$. For each set, we carried out runs at four values of sea quark mass in order to take the
TABLE II. Chirally extapolated parameters for $N_{f}=2$ lattices used in the heavy quark calculation. The lattice spacing is fixed by $\rho$ meson mass.

\begin{tabular}{lcc}
\hline \hline$\beta$ & $g_{\mathrm{MS}}^{2}(1 / a)$ & $a\left(\mathrm{GeV}^{-1}\right)$ \\
\hline 1.8 & 3.162 & $1.090(11)$ \\
1.95 & 2.812 & $0.7882(85)$ \\
2.1 & 2.562 & $0.559(11)$ \\
\hline
\end{tabular}

chiral limit of sea quark. The four sea quark masses are tuned so that the pseudoscalar-to-vector mass ratio $m_{P S} / m_{V}$ becomes roughly $0.80,0.75,0.70$, and 0.60 , which correspond to the range of quark mass of 3-0.5 times physical strange quark mass. The simulation parameters are listed in Table I, where the number of HMC trajectories is also shown. We note that at $\beta=2.1$ the configurations analyzed constitute the first half of the ensemble for each sea quark mass. The full set of configurations is used at $\beta=1.95$ and 1.8. The measurements are performed on configurations separated by 10 HMC trajectories at $\beta=1.8$ and 1.95 and by 5 trajectories at $\beta=2.1$. The statistical analysis is done using the jackknife method in order to take the correlation of successive trajectories into account. The bin size is 50 trajectories for all $N_{f}$ $=2$ runs, which has been determined to be a suitable length for eliminating autocorrelations [14].

The lattice spacing is determined for each $\beta$ value using the $\rho$ meson mass as input at the physical sea quark limit. The chiral extrapolation of light hadrons is discussed in Refs. $[28,29,14]$. The lattice spacings are listed in Table II.

In order to see the sea quark effect consistently using our choice of gauge and quark actions, we prepared ten sets of the quenched $\left(N_{f}=0\right)$ gauge configurations. The values of $\beta$ are chosen so that the string tension matches with each of full QCD configurations at $\beta=1.95$ or 2.1 at four sea quark masses and also in the chiral limit. For calculating lattice spacing and hence the physical value of the decay constants, the $\rho$ meson mass is used as input in conjunction with the vector masses measured on the lattice extrapolated to the light quark masses. The detail of our parameter choice in the quenched runs is summarized in Table III.

TABLE III. Simulation parameters for $N_{f}=0$. The lattice size employed is $16^{3} \times 32$ for $\beta=2.187$ -2.281 and $24^{3} \times 48$ for $\beta=2.416-2.575$. The lattice spacing is fixed by $\rho$ meson mass.

\begin{tabular}{ccccccccc}
\hline \hline$\beta$ & $c_{S W}$ & $g_{\overline{\mathrm{MS}}}^{2}(1 / a)$ & $a\left(\mathrm{GeV}^{-1}\right)$ & No. conf. & No. $K_{h}$ & No. $K_{l}$ & $K_{s}(K)^{-1}$ & $K_{s}(\phi)^{-1}$ \\
\hline 2.187 & 1.439 & 2.809 & $1.017(10)$ & 200 & 7 & 2 & $7.274(4)$ & $7.326(8)$ \\
2.214 & 1.431 & 2.767 & $0.966(10)$ & 200 & 7 & 2 & $7.293(4)$ & $7.340(8)$ \\
2.247 & 1.422 & 2.716 & $0.917(9)$ & 200 & 7 & 2 & $7.316(4)$ & $7.356(7)$ \\
2.281 & 1.412 & 2.664 & $0.896(10)$ & 220 & 7 & 2 & $7.348(4)$ & $7.395(8)$ \\
2.334 & 1.398 & 2.587 & $0.829(8)$ & 200 & 6 & 3 & $7.379(3)$ & $7.420(6)$ \\
2.416 & 1.378 & 2.477 & $0.734(9)$ & 190 & 8 & 2 & $7.415(4)$ & $7.452(7)$ \\
2.456 & 1.370 & 2.432 & $0.674(6)$ & 190 & 8 & 2 & $7.422(2)$ & $7.449(4)$ \\
2.487 & 1.363 & 2.401 & $0.652(7)$ & 200 & 8 & 2 & $7.434(3)$ & $7.462(5)$ \\
2.528 & 1.355 & 2.349 & $0.612(6)$ & 195 & 8 & 2 & $7.446(2)$ & $7.471(4)$ \\
2.575 & 1.345 & 2.298 & $0.574(6)$ & 200 & 8 & 3 & $7.458(2)$ & $7.480(4)$ \\
\hline \hline
\end{tabular}


TABLE IV. Smearing parameters used. The gauge-fixed smearing function takes the form $A \exp (-B r)$.

\begin{tabular}{lcccc}
\hline \hline$\beta$ & $N_{f}$ & $K_{\text {sea }}$ & $A$ & $B$ \\
\hline 1.8 & 2 & 0.1409 & 1.09 & 0.91 \\
1.8 & 2 & 0.1430 & 1.09 & 0.91 \\
1.8 & 2 & 0.1445 & 1.09 & 0.91 \\
1.8 & 2 & 0.1430 & 1.09 & 0.91 \\
1.95 & 2 & 0.1375 & 1.28 & 0.58 \\
1.95 & 2 & 0.139 & 1.28 & 0.58 \\
1.95 & 2 & 0.140 & 1.23 & 0.71 \\
1.95 & 2 & 0.141 & 1.27 & 0.6 \\
2.1 & 2 & 0.1357 & 1.28 & 0.54 \\
2.1 & 2 & 0.1367 & 1.28 & 0.54 \\
2.1 & 2 & 0.1374 & 1.28 & 0.54 \\
2.1 & 2 & 0.1382 & 1.28 & 0.54 \\
2.187 & 0 & - & 1.28 & 0.58 \\
2.214 & 0 & - & 1.28 & 0.58 \\
2.247 & 0 & - & 1.28 & 0.58 \\
2.281 & 0 & - & 1.28 & 0.58 \\
2.416 & 0 & - & 1.28 & 0.54 \\
2.456 & 0 & - & 1.28 & 0.54 \\
2.487 & 0 & - & 1.28 & 0.54 \\
2.528 & 0 & - & 1.28 & 0.54 \\
2.575 & 0 & - & 1.28 & 0.54 \\
\hline \hline
\end{tabular}

\section{B. Valence quarks}

The heavy and light quark propagators are calculated on each set of the gauge configurations for the $O(a)$-improved Wilson action with the same choice of $c_{S W}$ as used in the configuration generation. For each set of gauge configurations, eight values of the heavy quark mass are chosen so that their HQET mass (12) lie roughly on the interval of the $b$ and $c$ quark masses.

The light quark mass on the dynamical configurations is the same as their sea quark mass. In addition, we choose another quark mass for each set of configurations so that it satisfies $m_{P S} / m_{V}=0.688$. To compute any of the observables at the strange quark mass, the relevant observables are interpolated to the strange quark mass defined from the mass of the $K$ or $\phi$. The light quark masses for $N_{f}=0$ are chosen to take values approximately the same as those for the equivalent lattices (i.e., those lattices with matched string tension) for $N_{f}=2$.

The gauge configurations are fixed to the Coulomb gauge with a global maximum residue for $\operatorname{Tr}\left(\partial_{i} A_{i}\right)^{2}$ set to $10^{-14}$ or less. The light quark propagators are solved with local sources while the heavy quark propagators are computed with local and smeared sources. The smearing is made with the exponential function $A \exp (-B r)$, with the mean radius $1 / B$ chosen to approximately reproduce the heavy-light wave function. The parameters $A$ and $B$ are listed in Table IV.

Both light and heavy quark propagators are obtained with a solver based on the BiCGStab algorithm. For large values of heavy quark mass, stopping the solver if the residue becomes smaller than some minimum is not sufficient for obtaining the solution at large time separations. In this case, the iteration of the solver is applied a minimum of $2 \times T$ times, where $T$ is the temporal extent of the lattice, before applying the maximum residue criterion.

\section{Heavy-light current}

We compute the correlation functions constructed from the following operators:

$$
\begin{aligned}
P(x) & =\bar{l}(x) \gamma_{5} h(x), \\
A(x) & =\bar{l}(x) \gamma_{5} \gamma_{0} h(x), \\
\delta A(x) & =\bar{l}(x) \gamma_{5} \gamma_{0} \gamma \cdot \Delta h(x) .
\end{aligned}
$$

The heavy and light quark fields $h$ and $l$ are normalized with $\sqrt{1-3 K / 4 K_{c}}$, which is motivated with the nonrelativistic interpretation [12] together with the tadpole improvement [24]. The derivative current $\delta A$ is used to construct the modified current according to Eq. (15), and $\Delta$ is the discretised covariant derivative defined as

$$
\Delta_{i} h(x)=\frac{1}{2}\left[U_{i}(x) h(x+\hat{i})-U_{i}^{\dagger}(x-\hat{i}) h(x-\hat{i})\right] .
$$

Specifically, we measure the correlation functions

$$
\begin{array}{ll}
\sum_{\vec{x}}\left\langle P_{L}(\vec{x}, t) P_{S}^{\dagger}(0)\right\rangle, & \sum_{\vec{x}}\left\langle P_{L}(\vec{x}, t) P_{L}^{\dagger}(0)\right\rangle, \\
\sum_{\vec{x}}\left\langle A(\vec{x}, t) P_{S}^{\dagger}(0)\right\rangle, & \sum_{\vec{x}}\left\langle A(\vec{x}, t) P_{L}^{\dagger}(0)\right\rangle, \\
\sum_{\vec{x}}\left\langle\delta A(\vec{x}, t) P_{S}^{\dagger}(0)\right\rangle, & \sum_{\vec{x}}\left\langle\delta A(\vec{x}, t) P_{L}^{\dagger}(0)\right\rangle,
\end{array}
$$

where the subscripts $S$ and $L$ on the pseudoscalar operators indicate whether smeared or local operators are employed. The axial current $A$ and the derivative current are always local.

\section{ANALYSIS}

\section{A. Correlators}

The correlation functions defined above take the following form for large Euclidean time separation (we take $a=1$ for simplicity):

$$
\begin{aligned}
& \sum_{\vec{x}}\left\langle P_{L}(\vec{x}, t) P_{(L, S)}^{\dagger}(0)\right\rangle \\
&=\frac{\mathcal{Z}_{P_{L}} \mathcal{Z}_{P_{(L, S)}}}{2 M} e^{-M_{\text {pole }} T / 2} \cosh \left[M_{\text {pole }}(T / 2-t)\right] \\
&+\frac{\mathcal{Z}_{P_{L}}^{\prime} \mathcal{Z}_{P}^{\prime}}{2 M^{\prime}} e^{-M_{\text {pole }}^{\prime} T / 2} \cosh \left[M_{\text {pole }}^{\prime}(T / 2-t)\right],
\end{aligned}
$$




$$
\begin{aligned}
& \sum_{\vec{x}}\left\langle A(\vec{x}, t) P_{(L, S)}^{\dagger}(0)\right\rangle \\
& =\frac{\mathcal{Z}_{A} \mathcal{Z}_{P}}{2 M} e^{-M_{\text {pole }} T / 2} \sinh \left[M_{\text {pole }}(T / 2-t)\right] \\
& +\frac{\mathcal{Z}_{A}^{\prime} \mathcal{Z}_{P}^{\prime}}{2 M^{\prime}} e^{-M_{p o l e}^{\prime}}{ }^{T / 2} \sinh \left[M_{\text {pole }}^{\prime}(T / 2-t)\right], \\
& \sum_{\vec{x}}\left\langle\delta A(\vec{x}, t) P_{(L, S)}^{\dagger}(0)\right\rangle \\
& =\frac{\delta \mathcal{Z}_{A} \mathcal{Z}_{P_{(L, S)}}}{2 M} e^{-M_{\text {pole }} T / 2} \sinh \left[M_{\text {pole }}(T / 2-t)\right] \\
& +\frac{\delta \mathcal{Z}_{A}^{\prime} \mathcal{Z}_{P}^{\prime}}{2 M^{\prime}} e^{-M_{\text {pole }}^{\prime} T / 2} \sinh \left[M_{\text {pole }}^{\prime}(T / 2-t)\right]
\end{aligned}
$$

where $T$ is the temporal extent of the lattice, and $M$ and $M^{\prime}$ are masses of the ground and the first excited pseudoscalar states, respectively. The masses extracted from the $t$ dependence of the correlation functions are the pole masses, while the $M$ 's appearing in the denominator come from the normalization of states, and their definition need not be specified for calculating the combination of $f_{P} \sqrt{M}$.

The matrix elements $\mathcal{Z}$ are defined as

$$
\begin{gathered}
\mathcal{Z}_{P_{(L, S)}}=\left\langle 0\left|P_{(L, S)}(0)\right| P(\mathbf{0})\right\rangle, \\
\mathcal{Z}_{A}=\langle 0|A(0)| P(\mathbf{0})\rangle, \\
\delta \mathcal{Z}_{A}=\langle 0|\delta A(0)| P(\mathbf{0})\rangle,
\end{gathered}
$$

where $|P(\mathbf{0})\rangle$ represents the heavy-light pseudoscalar meson state at rest. The primed quantities are defined in a similar manner for the first excited state.

We carry out a simultaneous fit of the three correlators (21)-(23). Formally, for large Euclidean times, the contribution of the excited state will be negligible. However, it is included in the fit so as to use a wider range of Euclidean times and reduce the size of the statistical error. For those sets of configurations with lattice volumes of size $12^{3} \times 24$ and $16^{3} \times 32$ the sample size is large enough to perform a correlated fit of the local and smeared source data, where the correlation among different time slices are also taken into account. For the largest lattice $24^{3} \times 48$, such a correlation matrix appeared to be too large to achieve a stable fit with our statistics. We, therefore, use the uncorrelated fit throughout our statistical analysis, and check that the results are unchanged within statistical errors with the correlated fit when it is possible.

The fit criteria we apply for selecting the fit range are as follows [30]. (i) The quality of fit $Q$ should be acceptable, e.g., $Q>0.1$. (ii) The results for the chosen fit range should agree to within one standard deviation of the results when the minimum time slice is increased or decreased by one time slice. (iii) There should be agreement between the ground state results obtained using a single-exponential fit

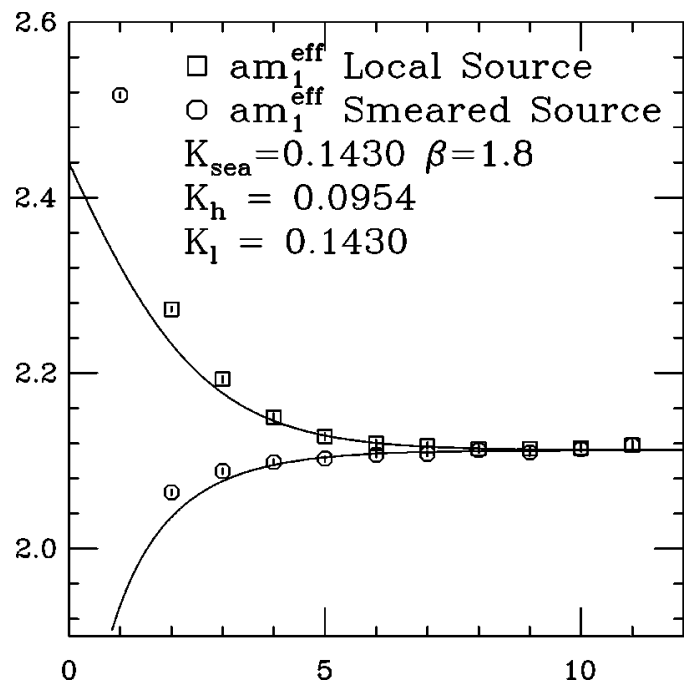

FIG. 1. Typical effective mass plots at $\beta=1.8$ for $N_{f}=2$. The fit range is from 3 to 11 .

and a double-exponential fit. This condition increases our confidence that higher state contamination is eliminated. (iv) In the double-exponential fit the ground and excited state energies must be statistically resolvable, i.e., there must be more than one standard deviation between their central values (since we expect the physical states to be distinctly separated).

The effective mass plots for the $\langle P P\rangle$ correlators, together with fit curves, are shown for a typical heavy-light meson mass in Figs. $1-3\left(N_{f}=2\right.$ case $)$ and in Figs. 4 and 5 (quenched case). For the $16^{3} \times 32$ lattices (Figs. 2 and 4), for which the correlated fit can be done, the $\chi^{2} / N_{\text {DF }}$ is also shown in the plots.

\section{B. Heavy-light decay constant}

The heavy-light decay constant $f_{P}$ is obtained through

$$
a^{3 / 2}\left(f_{P} \sqrt{M}\right)=Z_{A} \frac{1}{\sqrt{M}}\left(\mathcal{Z}_{A}-a d_{1} \delta \mathcal{Z}_{A}\right) .
$$

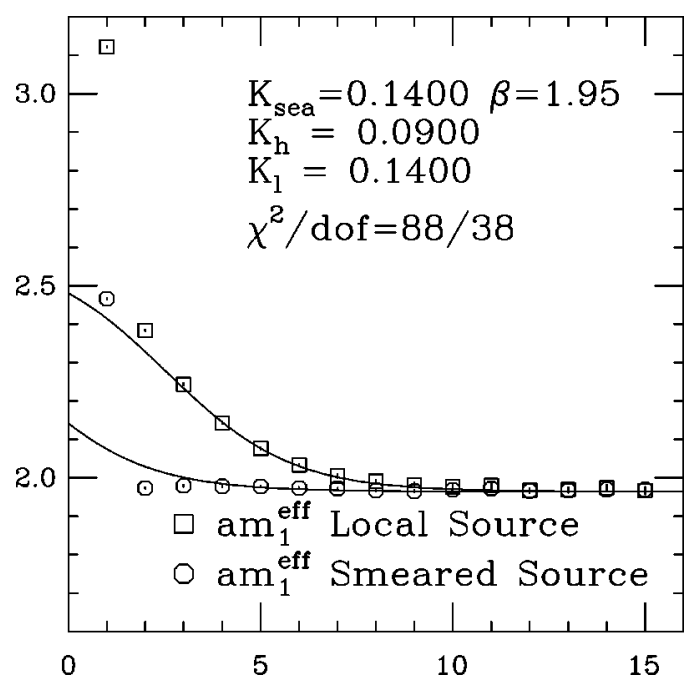

FIG. 2. Typical effective mass plots at $\beta=1.95$ for $N_{f}=2$. The fit range is from 4 to 15 . 


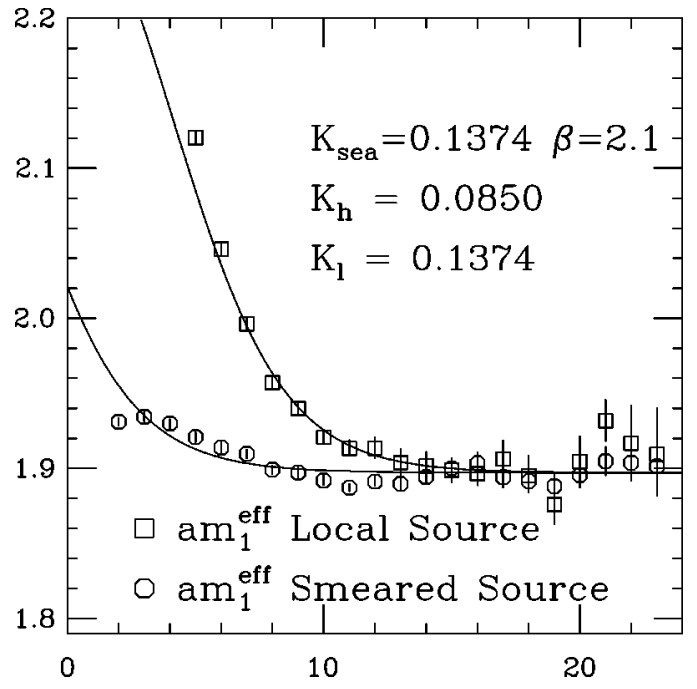

FIG. 3. Typical effective mass plots at $\beta=2.1$ for $N_{f}=2$. The fit range is from 5 to 21 .

In the massless limit, the renormalization constant $Z_{A}$ was previously calculated perturbatively to one-loop order for the RG-improved action [31]. Here we use a recent extension of this result to finite heavy quark masses made by Ishikawa et al. [32]. The results can be expressed in the form

$$
Z_{A}=1+\alpha_{s}\left[\rho_{A}+\frac{1}{\pi} \log \left(a m_{0}\right)\right]
$$

where the one-loop coefficient $\rho_{0}=\rho_{A}-(1 / \pi) \log \left(a m_{0}\right)$ is plotted in Fig. 6 as a function of $a m_{0}$, for the cases when $d_{1}$ takes the tree-level value and when it is ignored.

It is well known that perturbative expansions in lattice QCD are ill behaved when one uses the bare coupling constant $g_{0}^{2}$, and the use of some renormalized coupling defined through short-distance quantities gives a more convergent expansion [24]. Since a two-loop calculation of shortdistance quantities necessary to define an appropriate renor-

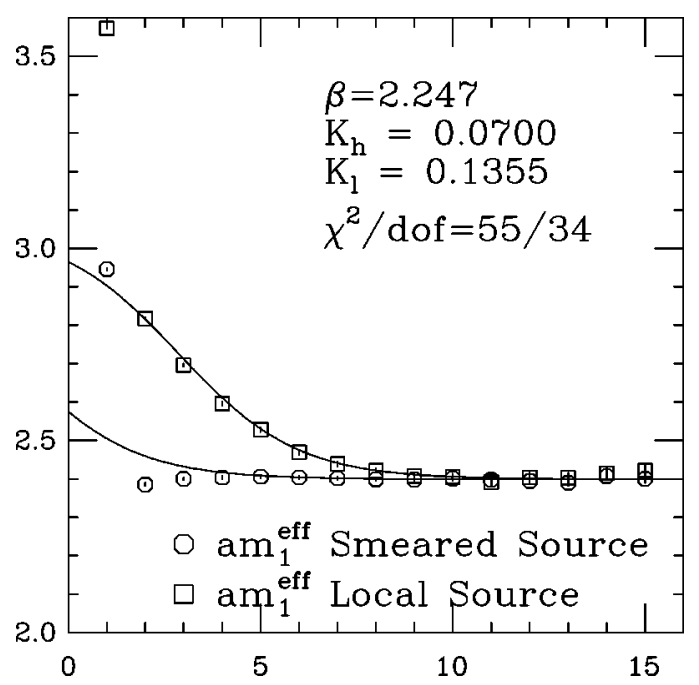

FIG. 4. Typical effective mass plots at $\beta=2.247$ for $N_{f}=0$. The fit range is from 4 to 14 .

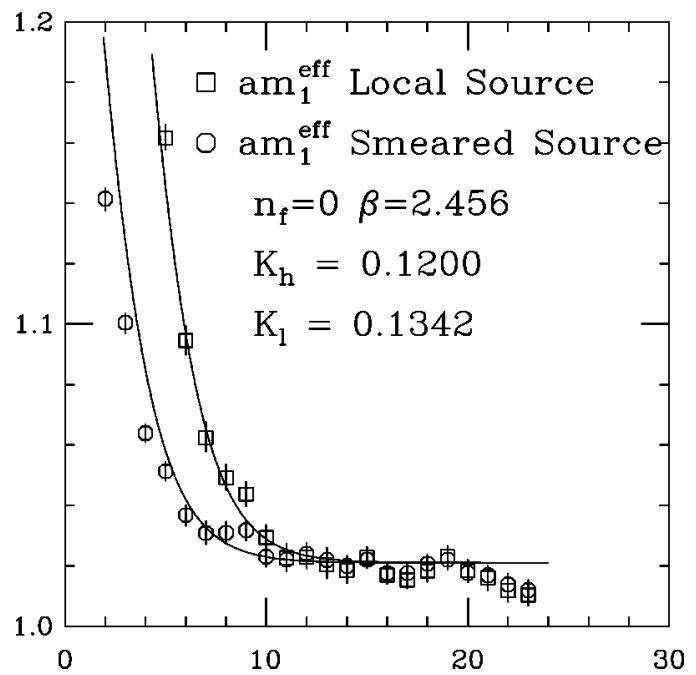

FIG. 5. Typical effective mass plots at $\beta=2.456$ for $N_{f}=0$. The fit range is from 5 to 21 .

malized coupling is not yet available for the RG gauge action, we use the continuum modified minimal subtraction scheme $(\overline{\mathrm{MS}})$ coupling as an alternative.

The one-loop perturbative relation between the bare and $(\overline{\mathrm{MS}})$ couplings for the RG improved gauge action and the $O(a)$-improved Wilson quark action is known as [31]

$$
\frac{1}{g_{\overline{\mathrm{MS}}}^{2}(\mu=1 / a)}=\frac{\beta}{6}+0.1000+0.0315 N_{f} .
$$

The tadpole improvement [24] may be applied to reduce the ultraviolet dominated pieces from the perturbative expansions by reorganizing the above relation as

$$
\frac{1}{g \frac{2}{\mathrm{MS}}(\mu=1 / a)}=\left(c_{0} P-8 c_{1} R\right) \frac{\beta}{6}-0.1006+0.0315 N_{f},
$$

where $P=\left\langle W_{1 \times 1}\right\rangle$ and $R=\left\langle W_{1 \times 2}\right\rangle$ are the expectation values of plaquette and $1 \times 2$ rectangle [13], and the one-loop expressions $P=1-0.1402 g^{2}$ and $R=1-0.2689 g^{2}$ are used to obtain the modified one-loop coefficient in Eq. (30). The values of $g_{\overline{\mathrm{MS}}}^{2}(\mu=1 / a)$ obtained with this formula are 3.162 , 2.812 , and 2.562 at $\beta=1.8,1.95$, and 2.1 , respectively, for the $N_{f}=2$ lattices. The same quantity for the quenched lattices is listed in Table III.

In Fig. 7 we plot $Z_{A}$ as a function of the bare heavy quark mass for the plaquette and RG-improved actions for an inverse lattice spacing of around $1.8 \mathrm{GeV}(\beta=5.9$ for the Wilson and $\beta=2.528$ for the RG action in the quenched approximation). In contrast to the large one-loop correction of order $-20 \%$ for the case of the plaquette gauge action, $Z_{A}$ is close to unity for the RG-improved action. 


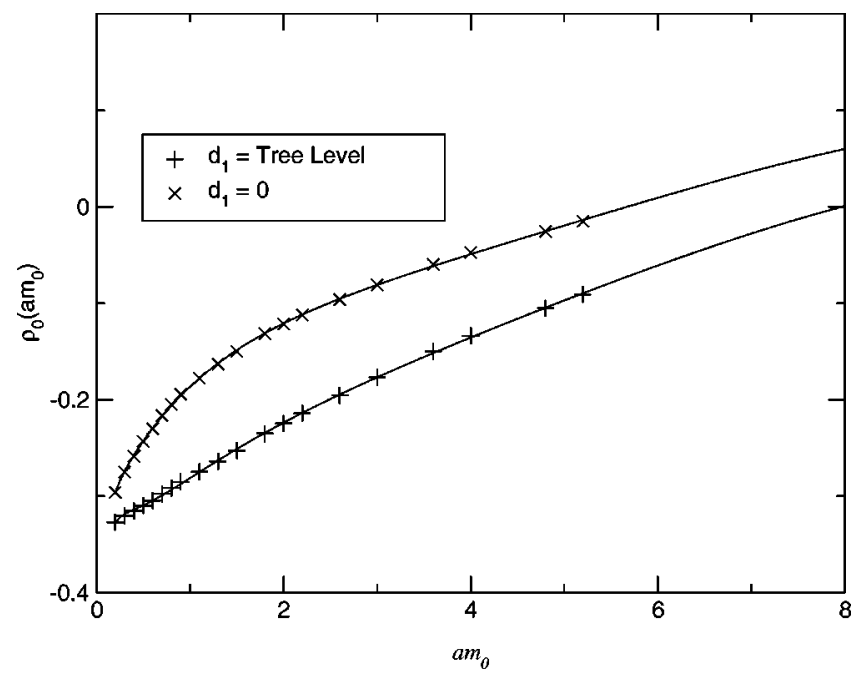

FIG. 6. The function $\rho_{0}\left(a m_{0}\right)$ where $Z_{A}\left(a m_{0}\right)=1$ $+\alpha_{s} \rho_{0}\left(a m_{0}\right)$. Pluses show results when the $1 / M$ correction to the current is included (i.e., $d_{1}$ takes its tree level value), and crosses are those without the correction $\left(d_{1}=0\right)$. Solid curves are interpolations.

\section{RESULTS}

\section{A. Effect of field rotation to the heavy-light current}

We first examine the effect of field rotation (13), which is reflected in Eqs. (15) and (27) as a correction proportional to $d_{1}$. An order counting suggests the size of the correction of the order of $a d_{1} \Lambda_{\mathrm{QCD}}$, which is about $d_{1} \times 15 \%$ at $1 / a \approx 2$ $\mathrm{GeV}$ if $\Lambda_{\mathrm{QCD}}=300 \mathrm{MeV}$. Since the tree-level coefficient $d_{1}$ given by Eq. (14) is smaller than 0.1 for any value of the bare quark mass $a m_{0}$, the size of the correction is naively estimated to be $O(2 \%)$.

In Fig. 8 we plot the quantity $f_{P}^{\text {rotated }} / f_{P}^{\text {unrotated }}-1$ as a function of the meson mass for $N_{f}=2$ and $N_{f}=0$, where $f_{P}^{\text {rotated }}$ includes the rotation term while it is ignored in

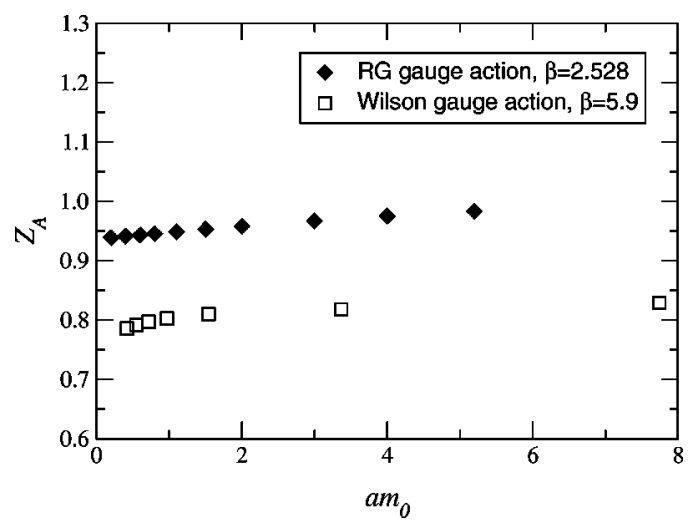

FIG. 7. The renormalization constant $Z_{A}$ as a function of $a m_{0}$ for the Wilson (at $\beta=5.9$ ) and $\mathrm{RG}$ (at $\beta=2.528$ ) gauge actions. In the case of the RG action, $Z_{A}$ is computed to specifically include the $1 / M$ correction to the current while in the Wilson action it is not. The inverse lattice spacing is roughly $1.8 \mathrm{GeV}$ (in the quenched approximation) for both cases.

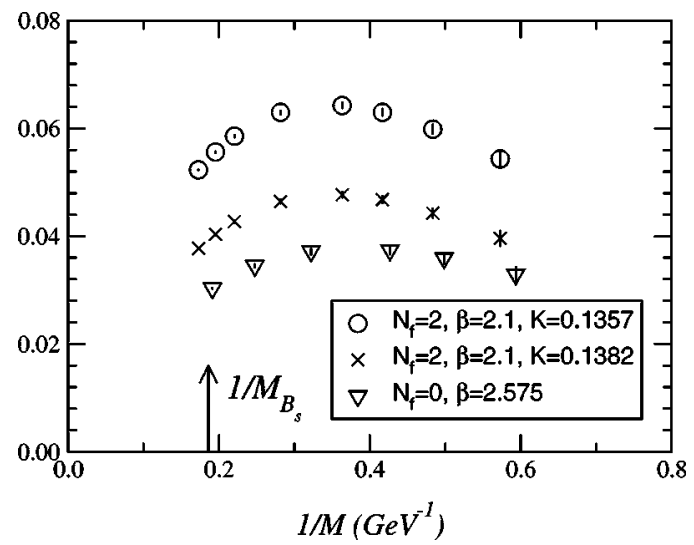

FIG. 8. Ratio $f_{P}^{\text {rotated }} / f_{P}^{\text {unrotated }}-1$ of decay constant including the current rotation to the lowest order current to that without the correction for $N_{f}=2$ (circles and crosses) and $N_{f}=0$ (triangles). The gauge couplings were picked so that the lattice spacing roughly matched with each other.

$f_{P}^{\text {unrotated }}$. Care must be exercised in this comparison to use the appropriate renormalization factors $Z_{A}$ for the rotated and unrotated currents shown in Fig. 6 since the diagram originating from the rotation term should be excluded for calculating $f_{P}^{\text {unrotated }}$. The lattice spacing for $\beta=2.575$ at $N_{f}=0$ is approximately equal to the lattice spacing, extrapolated to the chiral limit, for $\beta=2.1$ at $N_{f}=2$, which allows a more relevant comparison of the ratios. As one can see, the magnitude of correction is of the order of $3-7 \%$, which is larger than our expectation and cannot be ignored.

The large magnitude of this correction may partly originate from a power divergence of the matrix element of the higher dimensional operator $\delta A$ defined by Eq. (18), with which the naive order counting of $O\left(a \Lambda_{\mathrm{QCD}}\right)$ is changed to a size of $O(1)$. In principle this power divergence should be compensated by that in the perturbative matching. However, at the one-loop order in the calculation of Ref. [32], the compensation is incomplete.

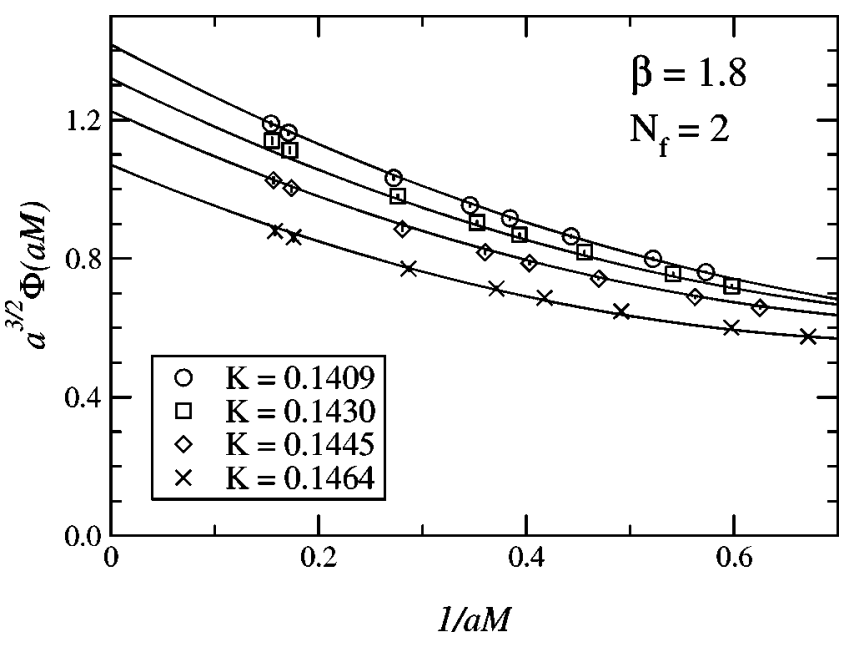

FIG. 9. A plot of $\Phi(a M)$ vs $1 / a M$ for $N_{f}=2$ at $\beta=1.8$. The data for four different sea quark masses are shown, and the light valence quark mass is set equal to the sea quark mass. 


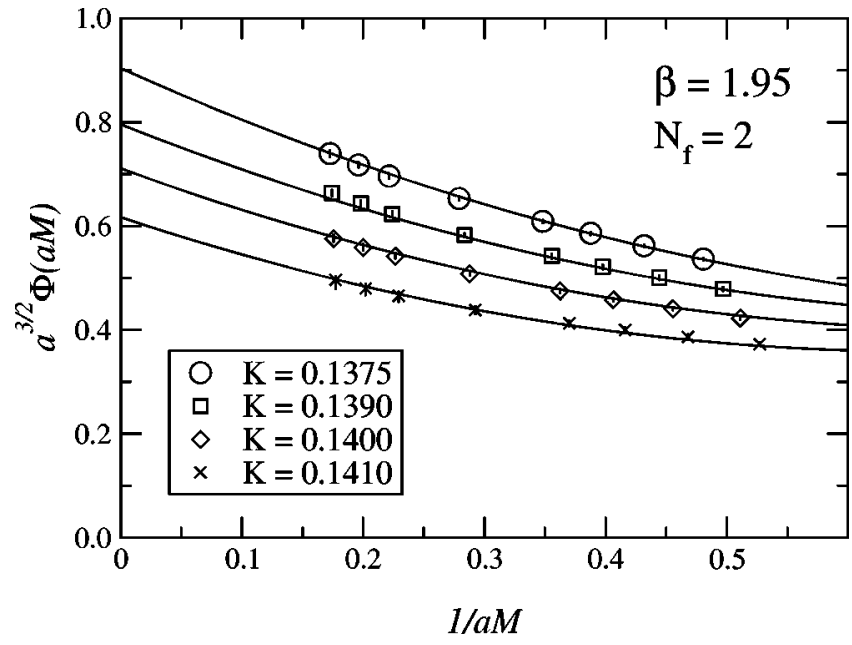

FIG. 10. Same as Fig. 9, but for $\beta=1.95$.

\section{B. Extrapolation to physical quark masses}

In order to obtain the heavy-light decay constant $f_{P} \sqrt{M}$ for the physical mass of $B_{(s)}$ and $D_{(s)}$ mesons, we fit the data with the following form:

$$
\begin{aligned}
a^{3 / 2} \Phi_{P}= & A_{0}+A_{1} a m_{q}+A_{2}\left(a m_{q}\right)^{2}+\frac{1}{a M}\left[B_{0}+B_{1} a m_{q}\right] \\
& +\frac{1}{(a M)^{2}} C_{0}
\end{aligned}
$$

where we define the renormalization group invariant decay constant $\Phi_{P}$ as

$$
a^{3 / 2} \Phi_{P}=\left(\frac{\alpha_{s}(M)}{\alpha_{s}\left(M_{B}\right)}\right)^{2 / \beta_{0}} a^{3 / 2}\left(f_{P} \sqrt{M}\right),
$$

with $\beta_{0}=11-\frac{2}{3} N_{f}$. The light quark mass is defined as $a m_{q}=\frac{1}{2}\left(1 / K-1 / K_{c}\right)$, where $K_{c}$ denotes the value at which pion mass made of sea quarks vanishes, and the HQET mass definition (12) is used for the heavy-light meson mass $M$.

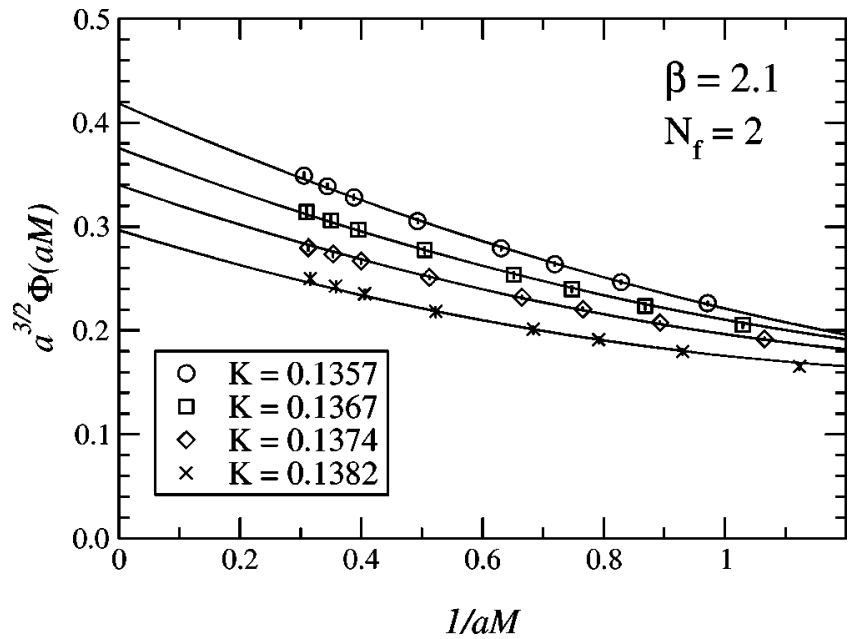

FIG. 11. Same as Fig. 9, but for $\beta=2.1$.

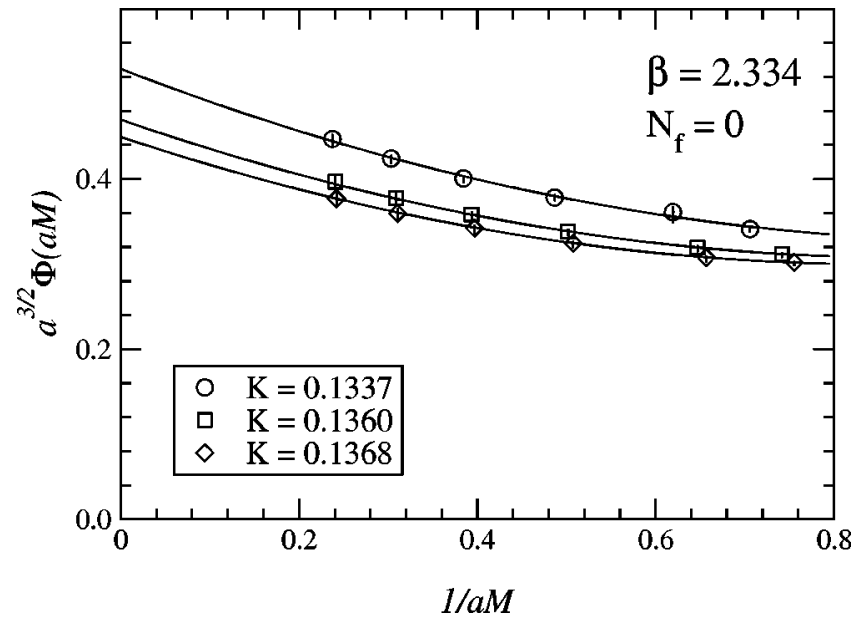

FIG. 12. A typical plot of $\Phi(a M)$ vs $1 / a M$ for $N_{f}=0$ at $\beta$ $=2.334$.

The renormalization group factor $\left[\alpha_{s}(M) / \alpha_{s}\left(M_{B}\right)\right]^{2 / \beta_{0}}$ is evaluated with a two-loop running coupling coefficient adopting $\Lambda_{\mathrm{QCD}}=300 \mathrm{MeV}$ for both $N_{f}=2$ and $N_{f}=0$. We have checked that the resulting decay constants are stable well within statistical errors under a variation of $\Lambda_{\mathrm{QCD}}$ by a factor 2 .

The form (31) is a truncated expansion of the matrix element in $1 / a M$ and in $a m_{q}$. It is possible to include higher order terms; however, the resulting fit coefficients are statistically not well determined, and we do not include such terms in our analyses.

In determining $f_{B_{d}}$ in the $N_{f}=2$ case, we only employ the matrix elements where the sea and valence light quark masses are matched. For $f_{B_{s}}$ we interpolate, at each sea quark mass, the matrix element in the valence light quark mass to the physical strange quark determined using the partially quenched analysis $[28,29,14]$. The values of the hopping parameter $K_{s}$ corresponding to the strange quark are

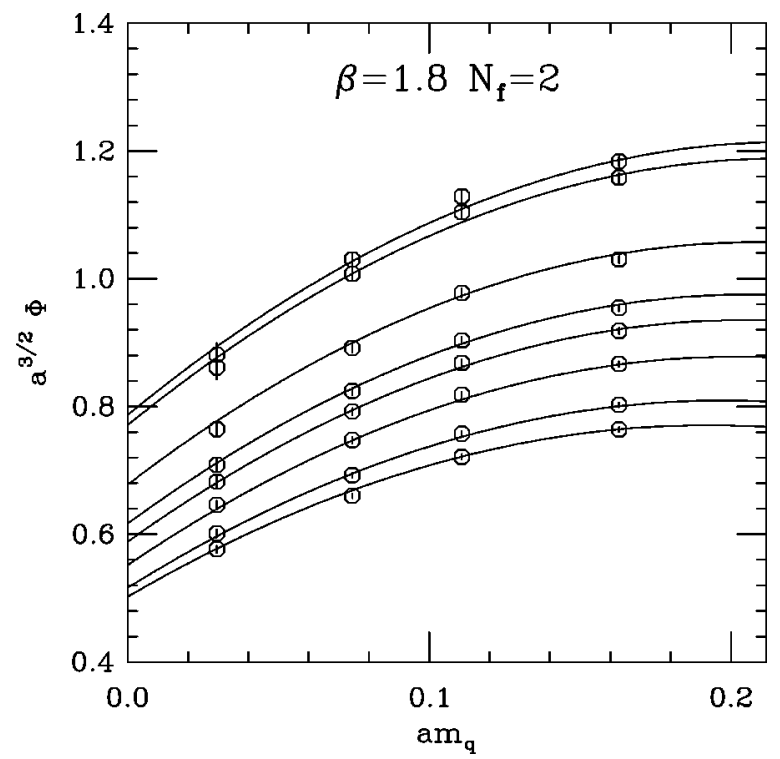

FIG. 13. A plot of $\Phi_{s}$ vs $a m_{q}$ for $\beta=1.80$ and $N_{f}=2$. 


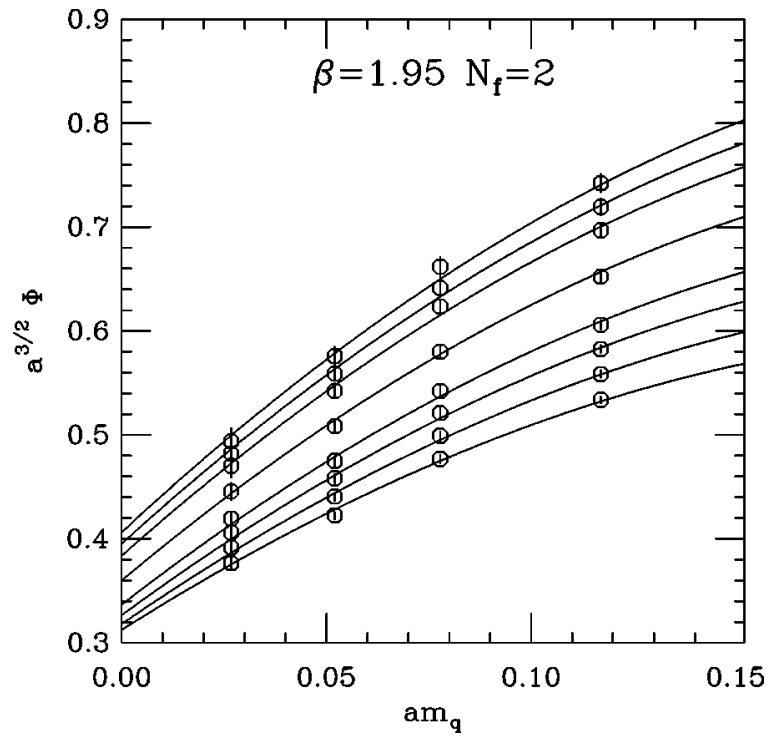

FIG. 14. A plot of $\Phi_{s}$ vs $a m_{q}$ for $\beta=1.95$ and $N_{f}=2$.

listed in Tables I and III, for the $K$ and $\phi$ meson masses as physical input. The critical hopping parameter $K_{c}$ necessary for evaluating the light quark mass $m_{q}$ is also listed in these tables.

The quenched data are analyzed with the same fit ansatz except that the term $A_{2}$ is set to zero, as the number of light quark masses in this case precluded a quadratic fit. For $f_{B s}$ and $f_{D s}$, the terms $A_{1}$ and $B_{1}$ are also set to zero, since there is no remaining light quark mass dependence once the strange quark mass is fixed.

Fits with the form (31) are represented in Figs. 9, 10, and 11 for three lattice spacing for $N_{f}=2$ and in Fig. 12 for $N_{f}$ $=0$. Data points and fit curves are plotted as a function of $1 / a M$ for fixed $a m_{q}$, from which one can see that the ansatz (31) represents the data quite well, except for a few points at $\beta=1.8$.

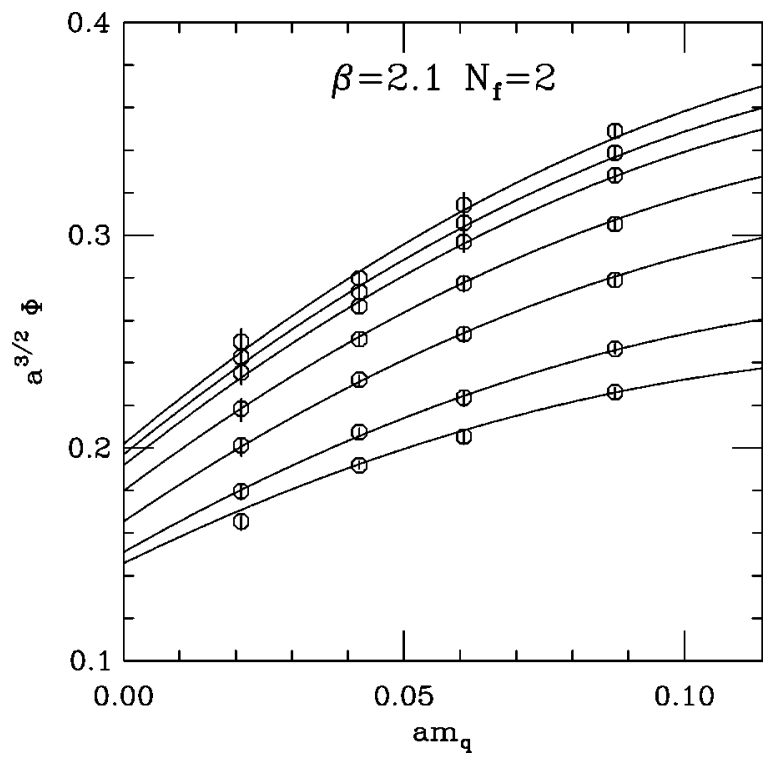

FIG. 15. A plot of $\Phi_{s}$ vs $a m_{q}$ for $\beta=2.10$ and $N_{f}=2$.

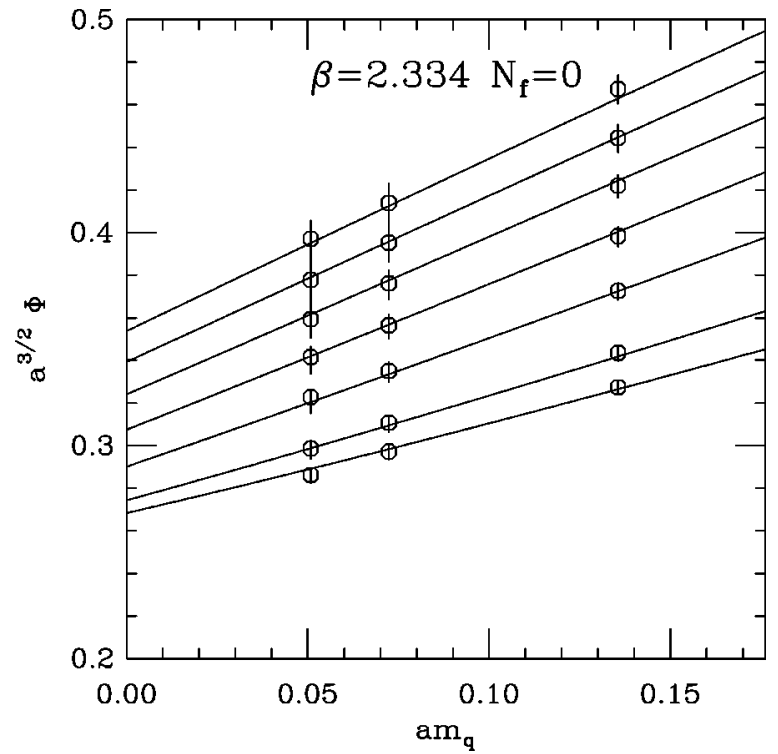

FIG. 16. A typical plot of $\Phi$ vs $a m_{q}$ for $N_{f}=0$. For $N_{f}=0, a m_{q}$ is the bare quark mass.

It is also illustrative to plot the data at fixed heavy quark masses as a function of $a m_{q}$, which is shown in Figs. 13-15 for $N_{f}=2$ and Fig. 16 for $N_{f}=0$. Since the results are given for fixed $K_{h}$, the heavy hopping parameter, we interpolated the curves for $a M$ as a function of $K_{h}$ and $a m_{q}$ and hence reexpressed the coefficients of Eq. (31) as a function of $K_{h}$ and $a m_{q}$. For $N_{f}=2$ we find clear curvature, which motivated us to introduce the term $A_{2}\left(a m_{q}\right)^{2}$ in Eq. (31). We, then, find good agreement of the fits to the data points. The fit parameters $A_{i}, B_{i}$, and $C_{0}$ are summarized in Tables $\mathrm{V}-\mathrm{X}$ for each set of configurations.

The $B$ and $D$ meson decay constants in physical units are obtained from Eq. (31) with their physical meson masses as input, and the numbers are summarized in Tables XI and XII, respectively. The lattice scale is set using the $\rho$ meson mass.

\section{Discretization effect}

The decay constants are plotted as a function of $a$ in Figs. $17\left(f_{B_{d}}\right), 18\left(f_{B_{s}}\right), 19\left(f_{D_{d}}\right)$, and $20\left(f_{D_{s}}\right)$. For $f_{B_{s}}$ (Fig. 18)

TABLE V. Chiral HQET fit parameters for $N_{f}=0$.

\begin{tabular}{cccccc}
\hline \hline$\beta$ & $A_{0}$ & $A_{1}$ & $B_{0}$ & $B_{1}$ & $C_{0}$ \\
\hline 2.187 & $0.670(27)$ & $1.10(6)$ & $-0.79(8)$ & $-1.17(9)$ & $0.485(60)$ \\
2.214 & $0.597(29)$ & $1.01(9)$ & $-0.66(8)$ & $-0.95(13)$ & $0.373(55)$ \\
2.247 & $0.556(25)$ & $1.00(8)$ & $-0.57(7)$ & $-0.88(11)$ & $0.296(51)$ \\
2.281 & $0.480(25)$ & $1.00(13)$ & $-0.43(6)$ & $-0.86(17)$ & $0.196(36)$ \\
2.334 & $0.412(22)$ & $0.93(10)$ & $-0.36(5)$ & $-0.78(14)$ & $0.173(31)$ \\
2.416 & $0.319(12)$ & $0.84(5)$ & $-0.20(2)$ & $-0.60(5)$ & $0.055(10)$ \\
2.456 & $0.317(2)$ & $0.52(24)$ & $-0.21(3)$ & $-0.31(22)$ & $0.065(7)$ \\
2.487 & $0.264(10)$ & $0.78(4)$ & $-0.16(2)$ & $-0.47(4)$ & $0.044(6)$ \\
2.528 & $0.237(16)$ & $0.82(34)$ & $-0.13(1)$ & $-0.50(23)$ & $0.032(4)$ \\
2.575 & $0.235(9)$ & $0.67(5)$ & $-0.14(1)$ & $-0.36(4)$ & $0.043(4)$ \\
\hline \hline
\end{tabular}


TABLE VI. Strange (defined from the $K$ ) HQET fit parameters for $N_{f}=0$.

\begin{tabular}{cccc}
\hline \hline$\beta$ & $A_{0}$ & $B_{0}$ & $C_{0}$ \\
\hline 2.187 & $0.785(23)$ & $-0.89(7)$ & $0.456(63)$ \\
2.214 & $0.699(22)$ & $-0.75(7)$ & $0.366(57)$ \\
2.247 & $0.650(20)$ & $-0.65(6)$ & $0.293(51)$ \\
2.281 & $0.583(15)$ & $-0.54(5)$ & $0.228(38)$ \\
2.334 & $0.492(13)$ & $-0.42(4)$ & $0.172(32)$ \\
2.416 & $0.365(15)$ & $-0.24(3)$ & $0.063(22)$ \\
2.456 & $0.357(10)$ & $-0.25(2)$ & $0.076(11)$ \\
2.487 & $0.315(8)$ & $-0.19(2)$ & $0.045(8)$ \\
2.528 & $0.288(12)$ & $-0.16(2)$ & $0.030(13)$ \\
2.575 & $0.273(7)$ & $-0.17(1)$ & $0.051(6)$ \\
\hline \hline
\end{tabular}

and $f_{D_{s}}$ (Fig. 20), we use the mass of $K$ to define the strange quark mass [as a short hand, we will refer to this as $f_{B_{s}}(K)$ and $\left.f_{D_{s}}(K)\right]$.

For the quenched data $\left(N_{f}=0\right)$, where ten data points are available, we observe a rapid decrease as the lattice spacing decreases from $a \approx 1 \mathrm{GeV}^{-1}$ to $a \approx 0.8 \mathrm{GeV}^{-1}$, followed by an almost constant behavior within statistical fluctuations below $a \approx 0.8 \mathrm{GeV}^{-1}$. We therefore fit the five data points for $a \lesssim 0.8 \mathrm{GeV}^{-1}$ as shown in the figures, and take this as our central value for the quenched result of the decay constant. From our data it is also possible that the decay constant is still decreasing in the region $a \lesssim 0.8 \mathrm{GeV}^{-1}$ toward the continuum limit and that the continuum result is about $10 \%$ lower than our estimate. This possibility can be accounted for by our estimate of systematic uncertainty as we discuss in the next section, where we consider systematic errors depending on $a$.

There is no evidence that $N_{f}=2$ data becomes independent of the lattice spacing. So we are not able to safely estimate the $B$ meson decay constant from our data. We may discuss, however, our results in the following way. The slope of the decrease of $f_{B}$ from $\beta=1.8$ to 1.95 quite resembles that for $N_{f}=0$ for stronger couplings, while the decrease from $\beta=1.95$ to 2.1 is somewhat reduced. If we suppose that the $N_{f}=2$ data behaves in a way similar to those for $N_{f}$

TABLE VII. Strange (defined from the $\phi$ ) HQET fit parameters for $N_{f}=0$.

\begin{tabular}{cccc}
\hline \hline$\beta$ & $A_{0}$ & $B_{0}$ & $C_{0}$ \\
\hline 2.187 & $0.814(22)$ & $-0.95(7)$ & $0.492(60)$ \\
2.214 & $0.723(20)$ & $-0.80(6)$ & $0.392(55)$ \\
2.247 & $0.667(20)$ & $-0.67(6)$ & $0.300(52)$ \\
2.281 & $0.611(15)$ & $-0.59(5)$ & $0.257(46)$ \\
2.334 & $0.512(13)$ & $-0.45(4)$ & $0.188(28)$ \\
2.416 & $0.380(13)$ & $-0.25(3)$ & $0.063(18)$ \\
2.456 & $0.362(7)$ & $-0.25(2)$ & $0.076(10)$ \\
2.487 & $0.326(8)$ & $-0.20(1)$ & $0.047(8)$ \\
2.528 & $0.301(20)$ & $-0.17(4)$ & $0.036(22)$ \\
2.575 & $0.282(6)$ & $-0.18(1)$ & $0.052(6)$ \\
\hline \hline
\end{tabular}

TABLE VIII. Chiral HQET fit parameters for $N_{f}=2$.

\begin{tabular}{ccccccc}
\hline \hline$\beta$ & $A_{0}$ & $A_{1}$ & $A_{2}$ & $B_{0}$ & $B_{1}$ & $C_{0}$ \\
\hline 1.80 & $0.95(2)$ & $0.48(4)$ & $-0.10(2)$ & $-1.27(6)$ & $-0.31(3)$ & $0.76(3)$ \\
1.95 & $0.51(2)$ & $0.61(6)$ & $-0.14(4)$ & $-0.72(8)$ & $-0.49(7)$ & $0.58(8)$ \\
2.10 & $0.25(1)$ & $0.56(9)$ & $-0.34(15)$ & $-0.18(1)$ & $-0.25(2)$ & $0.06(5)$ \\
\hline \hline
\end{tabular}

$=0$, the $N_{f}=2$ data would be already close to the asymptotic flattening at around $a=0.7 \mathrm{GeV}^{-1}$ and the data at $\beta=2.1$ may be taken as an estimate of the continuum limit. Since we cannot do better with the present data, we provisionally take the point at $\beta=2.1$ as the continuum value, allowing for the possibility that the true value may be somewhat smaller than our estimate. From the shape of the $\beta$ dependence of the $N_{f}=2$ data and their error bars, however, it seems likely that the continuum value is somewhat larger than that of $N_{f}=0$.

The extraction of the continuum limit for the $D$ meson decay constant is more subtle, since we see a larger drop from $\beta=1.95$ to $\beta=2.1$ rather than $\beta=1.8$ to 1.95 . While we take the data at $\beta=2.1$ as our provisional estimate for $f_{D}$, there is a possibility that the true value is smaller. Regardless, it can be concluded that the dynamical effect for $D$ mesons is appreciably smaller than that for $B$ mesons. We employ the same strategy as above for estimating the ratios $f_{B s} / f_{B d}$ and $f_{D s} / f_{D d}$ as shown in Figs. 21 and 22.

\section{Systematic errors}

We now examine the issue of systematic errors in our results for the decay constants. For this purpose we list the possible leading order errors and estimate their magnitude using naive power counting.

Generically these errors appear in three forms. First, we use tree-level mean-field estimates of the coefficients in the actions and currents and hence there will be radiative corrections, which are proportional to some power of $\alpha_{s}(\mu)$. Since the dominant part of the radiative corrections comes from a short distance region in the lattice four-momentum integral, we assume the scale $\mu$ to be $1 / a$. Secondly, discretization effects in the Lagrangians will be of the order of $(a|\mathbf{p}|)^{n}$, where $n$ is an integer and $\mathbf{p}$ is some soft momentum scale that characterizes the spatial momentum of the system. We take these soft modes to be of the order of $\Lambda_{\mathrm{QCD}}$. Finally, there are power corrections to the heavy quark effective Hamiltonian, which are of the order of some power of $\Lambda_{\mathrm{QCD}} / M$.

In detail we expect the following corrections in our case. (i) Gluon and light quark actions: For the RG-improved

TABLE IX. Strange (defined from the $K$ ) HQET fit parameters for $N_{f}=2$.

\begin{tabular}{ccccccc}
\hline \hline$\beta$ & $A_{0}$ & $A_{1}$ & $A_{2}$ & $B_{0}$ & $B_{1}$ & $C_{0}$ \\
\hline 1.80 & $1.09(2)$ & $2.57(28)$ & $-6.83(119)$ & $-1.43(4)$ & $-1.14(18)$ & $0.78(3)$ \\
1.95 & $0.60(2)$ & $2.58(37)$ & $-4.71(225)$ & $-0.79(7)$ & $-2.06(43)$ & $0.57(6)$ \\
2.10 & $0.30(1)$ & $1.32(36)$ & $-3.86(287)$ & $-0.20(1)$ & $-0.58(10)$ & $0.06(5)$ \\
\hline \hline
\end{tabular}


TABLE X. Strange (defined from the $\phi$ ) HQET fit parameters for $N_{f}=2$.

\begin{tabular}{ccccccc}
\hline \hline$\beta$ & $A_{0}$ & $A_{1}$ & $A_{2}$ & $B_{0}$ & $B_{1}$ & $C_{0}$ \\
\hline 1.80 & $1.13(2)$ & $2.64(26)$ & $-7.25(156)$ & $-1.49(3)$ & $-1.13(18)$ & $0.81(3)$ \\
1.95 & $0.62(3)$ & $2.65(37)$ & $-5.55(217)$ & $-0.82(8)$ & $-1.97(42)$ & $0.59(7)$ \\
2.10 & $0.31(1)$ & $1.22(36)$ & $-2.69(282)$ & $-0.21(1)$ & $-0.63(9)$ & $0.07(4)$ \\
\hline \hline
\end{tabular}

gauge action, the leading discretization error is of the same order as the plaquette gauge action, which is $\left(a \Lambda_{\mathrm{QCD}}\right)^{2}$. For the $O(a)$-improved Wilson quark action for light quarks, the leading error is of $O\left(\alpha_{s} a \Lambda_{\mathrm{QCD}}\right)$ since the coefficient $c_{S W}$ is tuned at one-loop level only by the mean field improvement. (ii) Heavy quark action: For the $O(a)$-improved Wilson quark action, the leading error appears in the $1 / M^{2}$ term at the tree level in the effective Hamiltonain, which is a source of systematic error of $O\left[\left(\Lambda_{\mathrm{QCD}} / M\right)^{2}\right]$. An additional error comes from the radiative correction that changes the relation between $M_{2}$ and $M_{B}$, and yields an uncertainty of $O\left(\alpha_{s} \Lambda_{\mathrm{QCD}} / M\right)$. (iii) Current corrections: The renormalization coefficient $Z_{A}$ is computed only to one-loop accuracy, hence higher order uncertainties are of the order of $\alpha_{s}^{2}$. Other corrections to the current are present, but these are of the same order as those in the heavy quark effective Hamiltonian.

The size of these corrections are estimated in Tables XIII and XIV for the $B$ and $D$ mesons. Numerical values are evaluated adopting the $\overline{\mathrm{MS}}$ coupling at the scale $\mu=1 / a \mathrm{de}-$ fined by Eq. (30) and $\Lambda_{\mathrm{QCD}}=300 \mathrm{MeV}$ or $\Lambda_{\mathrm{QCD}}=600 \mathrm{MeV}$, and substituting in $M$ the physical $B$ or $D$ meson mass. In the case of $N_{f}=0$, we only choose three representative $\beta$ values as the variation of the errors is so mild across the available range of lattice spacings. The total uncertainty is estimated by adding all individual sources in quadrature.

Since the estimates attempted in these tables are not more than an order counting exercise, the evaluated systematic errors may be underestimated. For instance, the typical momentum scale of the system can easily be twice as large as the QCD scale $\Lambda_{\mathrm{QCD}}=300 \mathrm{MeV}$. Therefore, we also make the similar estimates with $\Lambda_{\mathrm{QCD}}=600 \mathrm{MeV}$. In Fig. 23 we replot $f_{B_{d}}$ for $N_{f}=2$ as a function of lattice spacing. The statistical error is shown by thick error bars, while thin lines represent the total error for which the statistical and estimated systematic errors are added in quadrature. The systematic errors are estimated with $\Lambda_{\mathrm{QCD}}=300 \mathrm{MeV}$ (circles) or $\Lambda_{\mathrm{QCD}}=600 \mathrm{MeV}$ (diamonds). The systematic error is the smallest at the finest lattice spacing $(\beta=2.1)$ as one can see in Table XIII. This confirms our expectation that the result from this $\beta$ value provides the best estimate for $f_{B_{d}}$ in the continuum limit. It is also important to note that the data at coarser lattice spacings are consistent with the result at $\beta$ $=2.1$, if we take the systematic error into account with $\Lambda_{\mathrm{QCD}}=600 \mathrm{MeV}$. It suggests that our estimate of systematic errors is realistic for $\Lambda_{\mathrm{QCD}}=600 \mathrm{MeV}$, which we employ in quoting the systematic errors in the following.

The systematic errors can also be examined by taking other quantities to set the lattice scale, with which the systematic errors enter in a different way. To see this, instead of the rho meson mass employed in this work, we take the pion decay constant $f_{\pi}$ to normalize the decay constant, while the lattice scale from $m_{\rho}$ is used to fix the physical quark masses. The result is plotted in Fig. 24 for $f_{B_{d}}$ and in Fig. 25 for $f_{D_{d}}$. One can see that the lattice spacing dependence is rather milder when $f_{\pi}$ is used for normalization for both $N_{f}=2$ and $N_{f}=0$. Furthermore, with our estimation of the systematic error as presented above, the decay constants calculated with $f_{\pi}$ normalization are contained within the error band of those obtained with the $m_{\rho}$ normalization. It should be noted that lattice spacings determined using $f_{\pi}$ are larger than that determined from $m_{\rho}$. As a result, and this is particularly true at coarser lattice spacings, the perturbative corrections will be larger, as will the systematic error.

\section{E. Continuum estimate}

In Figs. 17-20 we plot our final results, including the estimated total error, for the continuum value of the heavy-

TABLE XI. Decay constants $f_{B d}$ and $f_{B s}$ at each bare gauge coupling.

\begin{tabular}{ccccccc}
\hline \hline$n_{f}$ & $\beta$ & $f_{B d}(\mathrm{GeV})$ & $f_{B s}(K)(\mathrm{GeV})$ & $f_{B s}(\phi)(\mathrm{GeV})$ & $f_{B s}(K) / f_{B d}$ & $f_{B s}(\phi) / f_{B d}$ \\
\hline 2 & 1.80 & $0.287(7)$ & $0.331(5)$ & $0.340(5)$ & $1.152(19)$ & $1.181(26)$ \\
2 & 1.95 & $0.234(8)$ & $0.276(7)$ & $0.283(8)$ & $1.179(43)$ & $1.211(45)$ \\
2 & 2.10 & $0.208(10)$ & $0.250(10)$ & $0.258(10)$ & $1.203(29)$ & $1.241(36)$ \\
0 & 2.187 & $0.229(7)$ & $0.268(5)$ & $0.276(5)$ & $1.171(13)$ & $1.121(16)$ \\
0 & 2.214 & $0.220(8)$ & $0.258(5)$ & $0.265(5)$ & $1.169(20)$ & $1.202(25)$ \\
0 & 2.247 & $0.223(6)$ & $0.260(5)$ & $0.266(5)$ & $1.165(16)$ & $1.194(19)$ \\
0 & 2.281 & $0.204(7)$ & $0.244(4)$ & $0.254(3)$ & $1.196(31)$ & $1.243(40)$ \\
0 & 2.334 & $0.195(7)$ & $0.232(4)$ & $0.240(4)$ & $1.186(29)$ & $1.227(35)$ \\
0 & 2.416 & $0.188(6)$ & $0.212(5)$ & $0.220(5)$ & $1.126(11)$ & $1.169(15)$ \\
0 & 2.456 & $0.204(11)$ & $0.228(4)$ & $0.232(3)$ & $1.111(58)$ & $1.103(39)$ \\
0 & 2.487 & $0.184(5)$ & $0.217(4)$ & $0.226(3)$ & $1.182(14)$ & $1.221(18)$ \\
0 & 2.528 & $0.182(11)$ & $0.219(6)$ & $0.228(9)$ & $1.190(104)$ & $1.235(130)$ \\
0 & 2.575 & $0.192(6)$ & $0.221(4)$ & $0.227(4)$ & $1.151(18)$ & $1.183(21)$ \\
\hline \hline
\end{tabular}


TABLE XII. Decay constants $f_{D d}$ and $f_{D s}$ at each bare gauge coupling.

\begin{tabular}{ccccccc}
\hline \hline$n_{f}$ & $\beta$ & $f_{D d}(\mathrm{GeV})$ & $f_{D s}(K)(\mathrm{GeV})$ & $f_{D s}(\phi)(\mathrm{GeV})$ & $f_{D s}(K) / f_{D d}$ & $f_{D s}(\phi) / f_{D d}$ \\
\hline 2 & 1.80 & $0.301(9)$ & $0.346(7)$ & $0.352(6)$ & $1.151(18)$ & $1.168(24)$ \\
2 & 1.95 & $0.284(13)$ & $0.312(11)$ & $0.316(11)$ & $1.097(40)$ & $1.111(39)$ \\
2 & 2.10 & $0.225(14)$ & $0.267(13)$ & $0.277(13)$ & $1.182(39)$ & $1.223(47)$ \\
0 & 2.187 & $0.258(4)$ & $0.295(3)$ & $0.300(3)$ & $1.143(7)$ & $1.165(8)$ \\
0 & 2.214 & $0.247(4)$ & $0.284(3)$ & $0.290(3)$ & $1.149(9)$ & $1.172(12)$ \\
0 & 2.247 & $0.250(4)$ & $0.287(3)$ & $0.293(3)$ & $1.146(8)$ & $1.169(10)$ \\
0 & 2.281 & $0.236(4)$ & $0.274(2)$ & $0.282(2)$ & $1.160(17)$ & $1.191(22)$ \\
0 & 2.334 & $0.229(4)$ & $0.263(3)$ & $0.269(3)$ & $1.148(12)$ & $1.175(14)$ \\
0 & 2.416 & $0.222(4)$ & $0.246(3)$ & $0.253(3)$ & $1.104(9)$ & $1.135(11)$ \\
0 & 2.456 & $0.229(8)$ & $0.254(3)$ & $0.257(3)$ & $1.129(73)$ & $1.119(46)$ \\
0 & 2.487 & $0.215(3)$ & $0.249(2)$ & $0.256(2)$ & $1.159(7)$ & $1.188(9)$ \\
0 & 2.528 & $0.215(10)$ & $0.250(4)$ & $0.256(6)$ & $1.133(67)$ & $1.159(81)$ \\
0 & 2.575 & $0.215(4)$ & $0.245(3)$ & $0.251(3)$ & $1.138(13)$ & $1.163(16)$ \\
\hline \hline
\end{tabular}

light decay constants at $a=0$. For the $N_{f}=2$ calculation with dynamical quarks, the central value is taken from the data at the finest lattice spacing $(\beta=2.1)$, and the total error shown is obtained by quadratically adding the statistical and systematic errors. Numerically, we find, for $N_{f}=2$,

$$
\begin{aligned}
f_{B d}^{N_{f}=2} & =208(10)(29) \mathrm{MeV} \\
f_{B s}^{N_{f}=2} & =250(10)(35)\left(\begin{array}{c}
+8 \\
-0
\end{array}\right) \mathrm{MeV}, \\
\left(\frac{f_{B s}}{f_{B d}}\right)^{N_{f}=2} & =1.203(29)(28)\left(\begin{array}{c}
+38 \\
-0
\end{array}\right), \\
f_{D d}^{N_{f}=2} & =225(14)(40) \mathrm{MeV}, \\
f_{D s}^{N_{f}=2} & =267(13)(48)\left(\begin{array}{c}
+10 \\
-0
\end{array}\right) \mathrm{MeV},
\end{aligned}
$$

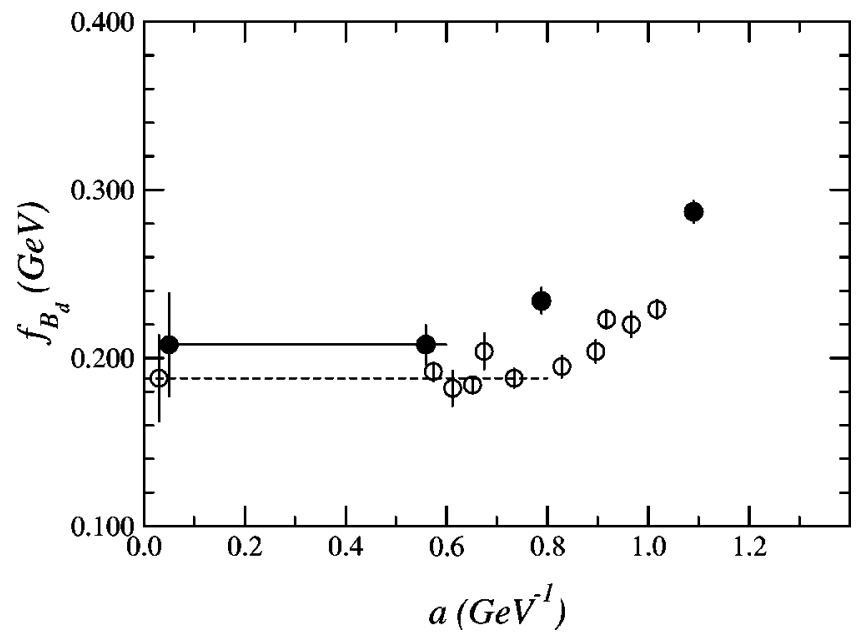

FIG. 17. $f_{B d}$ for $N_{f}=2$ (filled circles) and $N_{f}=0$ (open circles) as a function of lattice spacing $a$. The error bar for the data points represents the statistical errors only, while those in the continuum limit $(a=0)$ are the systematic and statistical errors added in quadrature.

$$
\left(\frac{f_{D s}}{f_{D d}}\right)^{N_{f}=2}=1.182(39)(25)\left(\begin{array}{c}
+41 \\
-0
\end{array}\right) .
$$

The first error is statistical, and the second error is the cumulative systematic error outlined above. For the ratios, as ambiguities due to the renormalization coefficient are eliminated, only the effect of the gluonic and light quark errors are included. In the case of those quantities involving the strange quark, the central value was taken from the strange quark mass defined from $m_{K}$, while a systematic error was estimated from mass of the $\phi$. If instead of adding the systematic errors quadratically, we added them linearly, the final results, taking $f_{B d}$ as an example, would be $f_{B d}$ $=208(10)(50) \mathrm{MeV}$. It is encouraging that our prediction for $f_{D_{s}}$ with $N_{f}=2$ is consistent with the recent experiments

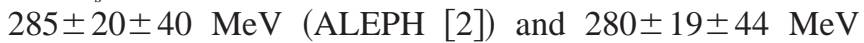
(CLEO [3]).

We also quote the results for the quenched case $N_{f}=0$, for which we employ a constant fit to the five data points in the region $a \lesssim 0.8 \mathrm{GeV}^{-1}$ corresponding to $\beta=2.575$

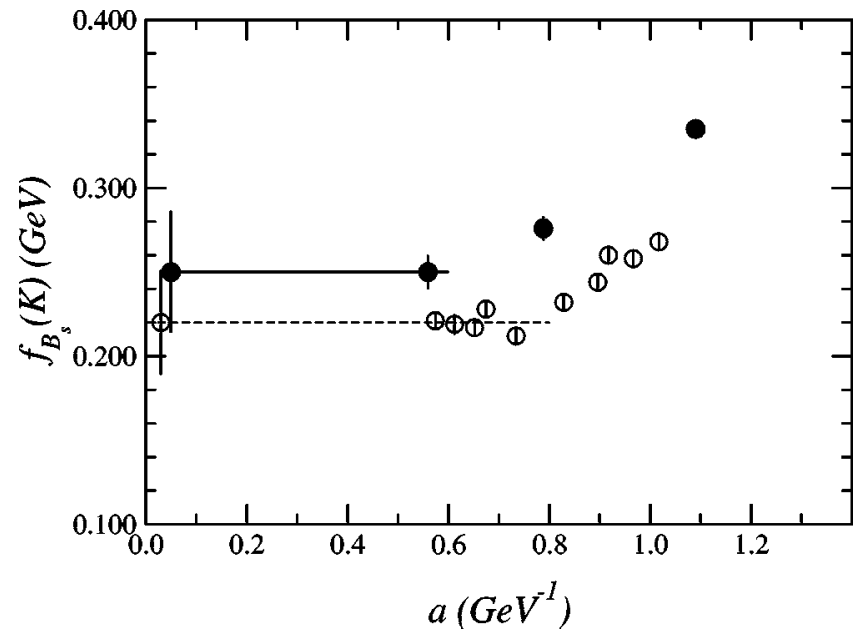

FIG. 18. Same as Fig. 17, but for $f_{B_{s}}(K)$. 


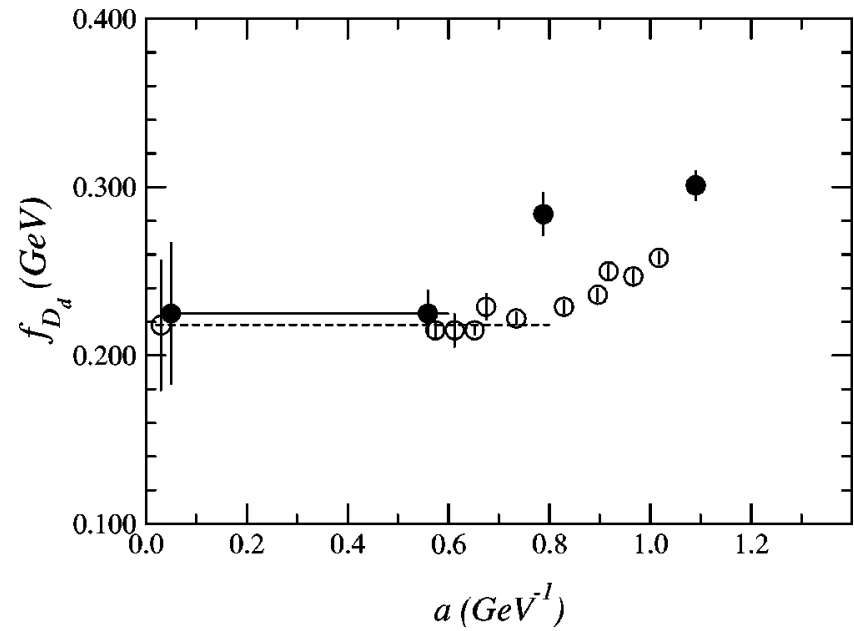

FIG. 19. Same as Fig. 17, but for $f_{D_{d}}$.

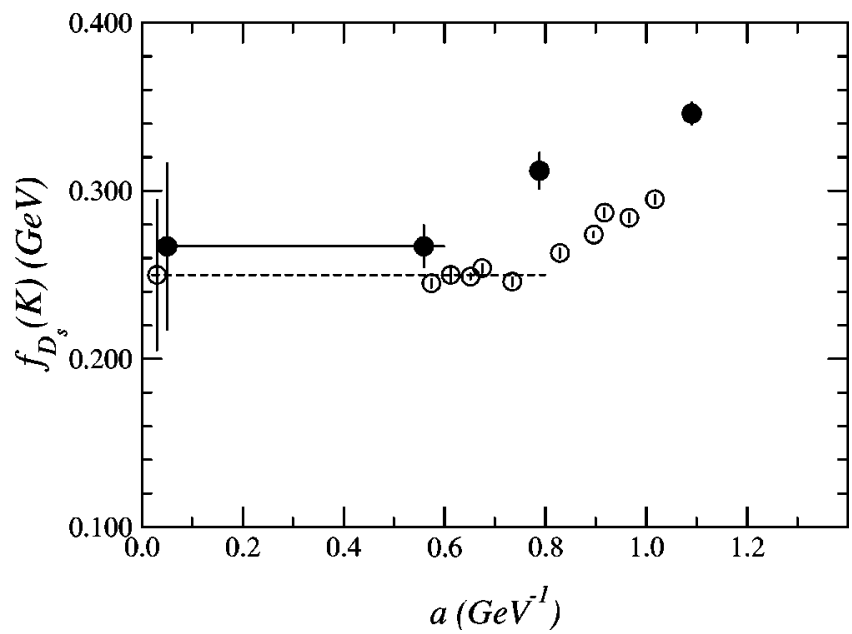

FIG. 20. Same as Fig. 17, but for $f_{D_{s}}(K)$.

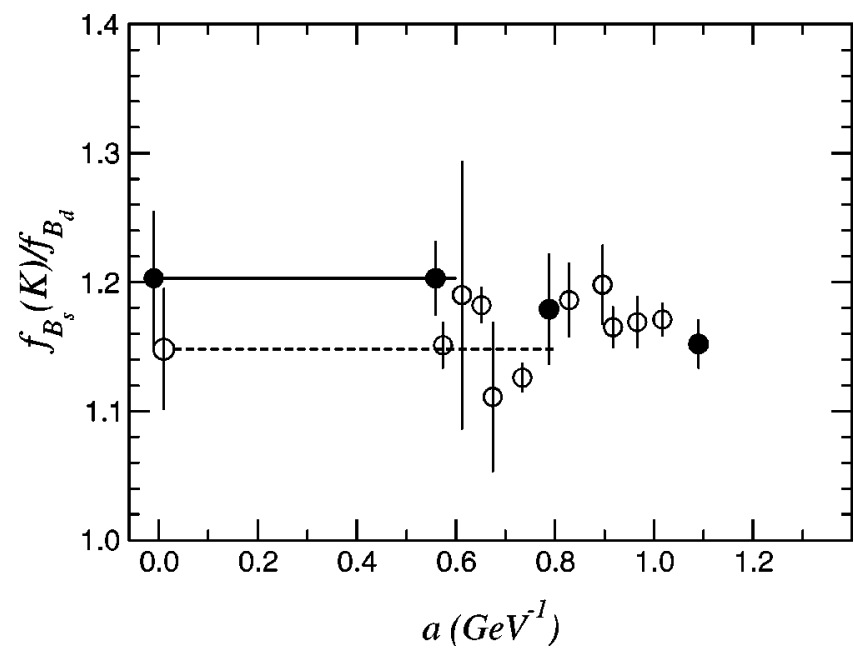

FIG. 21. A comparison of the ratio $f_{B s}(K) / f_{B d}$ for $N_{f}=0$ and $N_{f}=2$. The error bars of the continuum limit results are the systematic and statistical errors added in quadrature.

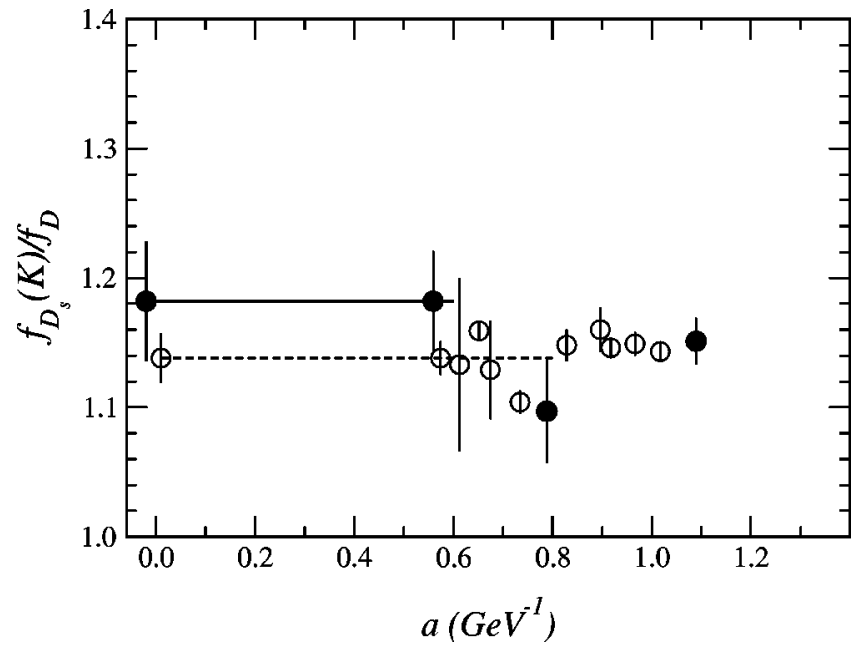

FIG. 22. Same as Fig. 21, but for $f_{D s}(K) / f_{D d}$.

-2.416 . The estimated systematic error varies only slightly in this region, and we find, for $N_{f}=0$,

$$
\begin{aligned}
f_{B d}^{N_{f}=0} & =188(3)(26) \mathrm{MeV}, \\
f_{B s}^{N_{f}=0} & =220(2)(31)\left(\begin{array}{c}
+8 \\
-0
\end{array}\right) \mathrm{MeV}, \\
\left(\frac{f_{B s}}{f_{B d}}\right)^{N_{f}=0} & =1.148(8)(20)\left(\begin{array}{c}
+39 \\
-0
\end{array}\right), \\
f_{D d}^{N_{f}=0} & =218(2)(39) \mathrm{MeV}, \\
f_{D s}^{N_{f}=0} & =250(1)(45)\left(\begin{array}{c}
+6 \\
-0
\end{array}\right) \mathrm{MeV},
\end{aligned}
$$

\begin{tabular}{|c|c|c|c|}
\hline \multicolumn{4}{|c|}{$\Lambda_{\mathrm{QCD}}=300 \mathrm{MeV}$} \\
\hline$\beta$ & 1.8 & 1.95 & 2.1 \\
\hline$\left(\Lambda_{\mathrm{QCD}} / M_{B}\right)^{2},\left(\Lambda_{\mathrm{QCD}} / M_{D}\right)^{2}$ & $<1 \%, 3 \%$ & $<1 \%, 3 \%$ & $<1 \%, 3 \%$ \\
\hline$\alpha_{s}^{2}$ & $6 \%$ & $5 \%$ & $4 \%$ \\
\hline$\left(a \Lambda_{\mathrm{QCD}}\right)^{2}$ & $11 \%$ & $6 \%$ & $3 \%$ \\
\hline$\alpha_{s} a \Lambda_{\mathrm{QCD}}$ & $8 \%$ & $5 \%$ & $3 \%$ \\
\hline$\alpha_{s} \Lambda_{\mathrm{QCD}} / M_{B}, \alpha_{s} \Lambda_{\mathrm{QCD}} / M_{D}$ & $1 \%, 4 \%$ & $1 \%, 4 \%$ & $1 \%, 3 \%$ \\
\hline total & $15 \%, 16 \%$ & $9 \%, 11 \%$ & $6 \%, 7 \%$ \\
\hline \multicolumn{4}{|c|}{$\Lambda_{\mathrm{QCD}}=600 \mathrm{MeV}$} \\
\hline$\beta$ & 1.8 & 1.95 & 2.1 \\
\hline$\left(\Lambda_{\mathrm{QCD}} / M_{B}\right)^{2},\left(\Lambda_{\mathrm{QCD}} / M_{D}\right)^{2}$ & $1 \%, 10 \%$ & $1 \%, 10 \%$ & $1 \%, 10 \%$ \\
\hline$\alpha_{s}^{2}$ & $6 \%$ & $5 \%$ & $4 \%$ \\
\hline$\left(a \Lambda_{\mathrm{QCD}}\right)^{2}$ & $42 \%$ & $22 \%$ & $11 \%$ \\
\hline$\alpha_{s} a \Lambda_{\mathrm{QCD}}$ & $16 \%$ & $11 \%$ & $7 \%$ \\
\hline$\alpha_{s} \Lambda_{\mathrm{QCD}} / M_{B}, \alpha_{s} \Lambda_{\mathrm{QCD}} / M_{D}$ & $3 \%, 7 \%$ & $3 \%, 7 \%$ & $2 \%, 6 \%$ \\
\hline total & $45 \%, 47 \%$ & $25 \%, 28 \%$ & $14 \%, 18 \%$ \\
\hline
\end{tabular}

TABLE XIII. Estimates of systematic errors for $N_{f}=2 . \Lambda_{\mathrm{QCD}}$ is taken to be $300 \mathrm{MeV}$ (top half) or $600 \mathrm{MeV}$ (bottom half). 
TABLE XIV. Estimate of systematic errors for $N_{f}=0 . \Lambda_{\mathrm{QCD}}$ is taken to be $300 \mathrm{MeV}$ (top half) or $600 \mathrm{MeV}$ (bottom half).

\begin{tabular}{|c|c|c|c|}
\hline \multicolumn{4}{|c|}{$\Lambda_{Q C D}=300 \mathrm{MeV}$} \\
\hline$\beta$ & 2.187 & 2.416 & 2.575 \\
\hline$\left(\Lambda_{\mathrm{QCD}} / M_{B}\right)^{2},\left(\Lambda_{\mathrm{QCD}} / M_{D}\right)^{2}$ & $<1 \%, 3 \%$ & $<1 \%, 3 \%$ & $<1 \%, 3 \%$ \\
\hline$\alpha_{s}^{2}$ & $5 \%$ & $4 \%$ & $3 \%$ \\
\hline$\left(a \Lambda_{\mathrm{QCD}}\right)^{2}$ & $9 \%$ & $5 \%$ & $3 \%$ \\
\hline$\alpha_{s} a \Lambda_{\mathrm{QCD}}$ & $7 \%$ & $4 \%$ & $3 \%$ \\
\hline$\alpha_{s} \Lambda_{\mathrm{QCD}} / M_{B}, \alpha_{s} \Lambda_{\mathrm{QCD}} / M_{D}$ & $1 \%, 4 \%$ & $1 \%, 3 \%$ & $1 \%, 3 \%$ \\
\hline total & $13 \%, 13 \%$ & $8 \%, 9 \%$ & $5 \%, 7 \%$ \\
\hline \multicolumn{4}{|c|}{$\Lambda_{\mathrm{QCD}}=600 \mathrm{MeV}$} \\
\hline$\beta$ & 2.187 & 2.416 & 2.575 \\
\hline$\left(\Lambda_{\mathrm{QCD}} / M_{B}\right)^{2},\left(\Lambda_{\mathrm{QCD}} / M_{D}\right)^{2}$ & $1 \%, 10 \%$ & $1 \%, 10 \%$ & $1 \%, 10 \%$ \\
\hline$\alpha_{s}^{2}$ & $5 \%$ & $4 \%$ & $3 \%$ \\
\hline$\left(a \Lambda_{\mathrm{QCD}}\right)^{2}$ & $37 \%$ & $19 \%$ & $12 \%$ \\
\hline$\alpha_{s} a \Lambda_{\mathrm{QCD}}$ & $14 \%$ & $9 \%$ & $6 \%$ \\
\hline$\alpha_{s} \Lambda_{\mathrm{QCD}} / M_{B}, \alpha_{s} \Lambda_{\mathrm{QCD}} / M_{D}$ & $3 \%, 7 \%$ & $2 \%, 6 \%$ & $2 \%, 6 \%$ \\
\hline total (linear) & $60 \%, 73 \%$ & $35 \%, 48 \%$ & $24 \%, 37 \%$ \\
\hline total (quadratic) & $40 \%, 42 \%$ & $22 \%, 24 \%$ & $14 \%, 18 \%$ \\
\hline
\end{tabular}

$$
\left(\frac{f_{D s}}{f_{D d}}\right)^{N_{f}=0}=1.138(5)(18)\left(\begin{array}{c}
+29 \\
-0
\end{array}\right)
$$

where the systematic errors are assigned with the same strategy as for the case of $N_{f}=2$.

These quenched decay constants lie at the upper end when compared with those from previous quenched lattice calculations, whose recent summary is $f_{B d}=170(20) \mathrm{MeV}, f_{B s}$ $=195(20) \mathrm{MeV}$ [4], and $f_{D d}=200(20) \mathrm{MeV}, f_{D s}$ $=220\left({ }_{-20}^{+25}\right) \mathrm{MeV}$ [5]. However, taking the systematic errors

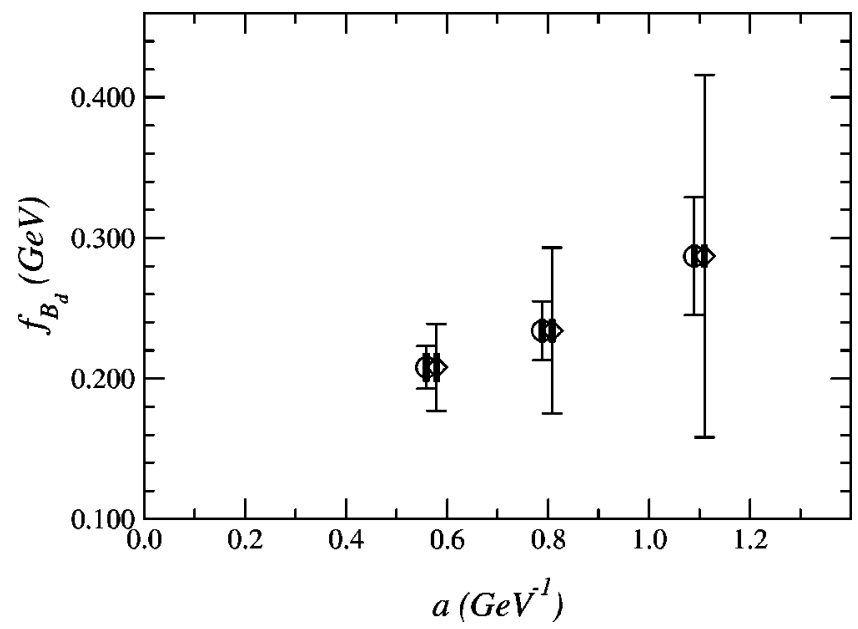

FIG. 23. $f_{B_{d}}$ with combined statistical and systematic errors for $N_{f}=2$. The statistical error is shown by thick error bars, while thin lines represent the total error for which the statistical and estimated systematic errors are added in quadrature. Circles employ $\Lambda_{\mathrm{QCD}}$ $=300 \mathrm{MeV}$ for estimating systematic errors, and diamonds $\Lambda_{\mathrm{QCD}}$ $=600 \mathrm{MeV}$.

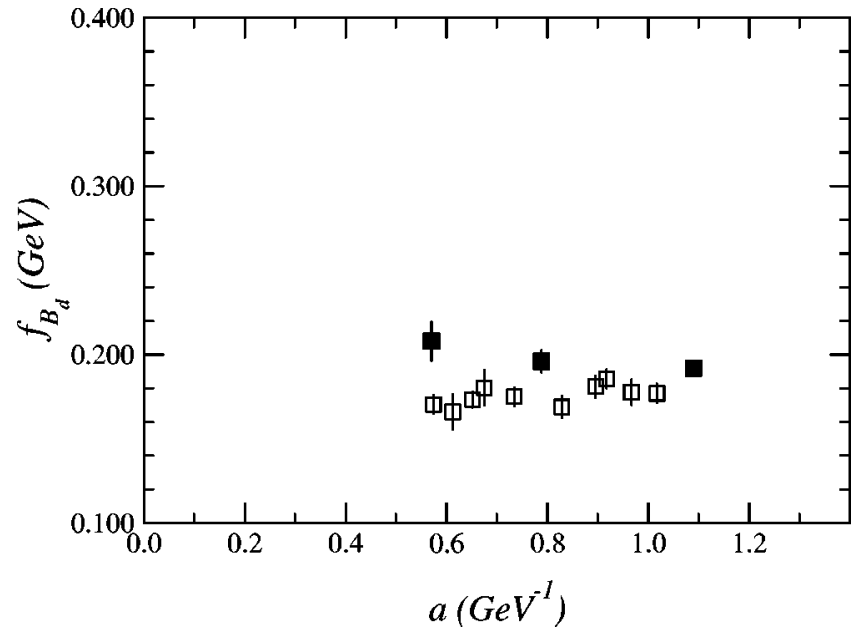

FIG. 24. $f_{B d}$ for $N_{f}=2$ (filled squares) and $N_{f}=0$ (open squares) as a function of lattice spacing $a . f_{B d}$ is normalized using $f_{\pi}$. The error bar for the data points represents the statistical errors only. The systematic error will be roughly the same size as that for Fig. 23.

in our results into account, our quenched results with the RG-improved action are consistent with the previous data obtained with the plaquette gauge action.

\section{F. Quenching effects}

In order to see the effect of introducing sea quarks it is instructive to take the ratio of the results for $N_{f}=2$ and $N_{f}$ $=0$, for which we find

$$
\frac{f_{B d}^{N_{f}=2}}{f_{B d}^{N_{f}=0}}=1.11(6),
$$

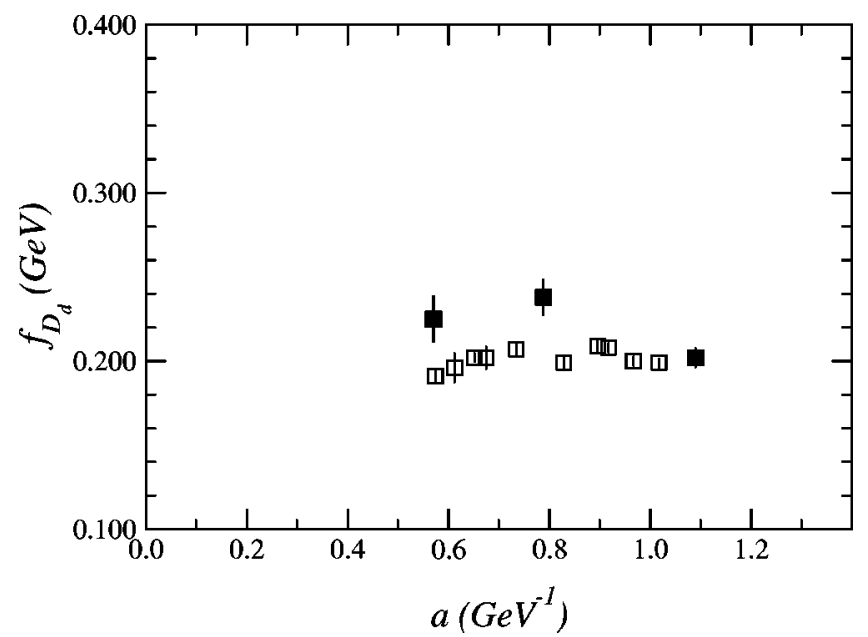

FIG. 25. $f_{D d}$ for $N_{f}=2$ (filled squares) and $N_{f}=0$ (open squares) as a function of lattice spacing $a . f_{D d}$ is normalized using $f_{\pi}$. The error bar for the data points represents the statistical errors only. The systematic error will be roughly the same size as that for Fig. 23. 


$$
\begin{gathered}
\frac{f_{B s}^{N_{f}=2}}{f_{B s}^{N_{f}=0}}=1.14(5), \\
\left(\frac{f_{B s}}{f_{B d}}\right)^{N_{f}=2} /\left(\frac{f_{B s}}{f_{B d}}\right)^{N_{f}=0}=1.05(3), \\
\frac{f_{D d}^{N_{f}=2}}{f_{D d}^{N_{f}=0}}=1.03(6), \\
\frac{f_{D s}^{N_{f}=2}}{f_{D s}^{N_{f}=0}}=1.07(5), \\
\left(\frac{f_{D s}}{f_{D d}}\right)^{N_{f}=2} /\left(\frac{f_{D s}}{f_{D d}}\right)^{N_{f}=0}=1.04(3) .
\end{gathered}
$$

The errors quoted above are statistical only. We observe that the central value increases by $10-15 \%$ for the $B$ meson decay constants when two flavors of dynamical quarks are introduced, which has statistical significance of 2 to 3 standard deviations. For the $D$ meson decay constant, on the other hand, the observed increase is only $3-7 \%$, and the effect is statistically not very significant. For the ratio of decay constants we find only a small change from $N_{f}=0$ to $N_{f}=2$.

We assumed that the systematic errors cancel in the ratio. This assumption is supported by the similar $a$ dependence of $f_{B}$ for $N_{f}=2$ and for $N_{f}=0$. To be convincing, however, more data is necessary especially in the smaller $a$ region.

An increase of $B$ meson decay constants in the presence of dynamical sea quarks has already been suggested in Refs. $[9,10]$. Our results also show this trend, providing further evidence that the upward shift is real.

\section{CONCLUSIONS}

In this paper we have presented a calculation of the heavy-light axial decay constants $f_{B d}, f_{B s}, f_{D d}, f_{D s}$ and their ratios in lattice QCD with two degenerate flavors of sea quark $\left(N_{f}=2\right)$ where the same discretization scheme has been employed for the sea and light valence quarks. In order to carry out the calculation with the computational resources available, the heavy quarks are treated using an effective field theory approach, and the light quark and gluon fields actions are improved to minimize the discretization error. The calculation is also made in the quenched $\left(N_{f}=0\right)$ case since these decay constants have not been calculated before with this combination of actions.

In comparing our $N_{f}=0$ and $N_{f}=2$ results we see that $f_{B d}$ and $f_{B s}$ for $N_{f}=2$ are significantly larger than the $N_{f}$ $=0$ results by $2-3$ standard deviations, indicating a shift of $10-15 \%$. On the other hand, the same cannot be said for the decay constants $f_{D d}$ and $f_{D s}$. It is encouraging that our prediction $f_{D_{s}}=267\left({ }_{-50}^{+51}\right) \mathrm{MeV}$ with $N_{f}=2$, where the total error is obtained by quadrature, is consistent with recent experiments. In conjunction with the available experimental data, our values for the $N_{f}=2 B$ meson decay constants $f_{B_{d}}=208(31) \mathrm{MeV}$ and $f_{B_{s}}=250\left({ }_{-36}^{+37}\right) \mathrm{MeV}$ are consistent with the hypothesis that the Wolfenstein parameter $\rho$ [33] is positive. Given our results for $N_{f}=0$ and $N_{f}=2$, it is reasonable to think that additional flavors of sea quarks will increase $f_{B d}$ and $f_{B s}$ still further, which in turn favors a positive value for $\rho$ even more.

The unsatisfactory aspect of our results is a sizable variation of the decay constants with lattice spacing. A possible origin of this problem is a necessity to include $O(a)$ and higher improvement terms in the axial vector current. Higher order corrections in the renormalization constants may also be important at the coarse lattice spacings of $a^{-1} \approx 1-2$ $\mathrm{GeV}$ explored in the present simulation. The study of these issues is clearly needed to consolidate the results for $N_{f}=2$ and further explore the final goal of predicting the heavylight decay constants for the realistic spectrum of dynamical sea quarks.

\section{ACKNOWLEDGMENTS}

The authors would like to express their thanks to K.-I. Ishikawa for providing expressions for the axial renormalization coefficient. This work is supported in part by the Grantsin-Aid of Ministry of Education (Nos. 09304029, 10640246, 10640248, 10740107, 11640250, 11640294, 11740162). T.M. and A.A.K. were supported by the JSPS Research for the Future Program (Project No. JSPS-RFTF 97P01102).
[1] A.J. Buras, M. Jamin, and P.H. Weisz, Nucl. Phys. B347, 491 (1990).

[2] ALEPH Collaboration, contributed paper to XXXth International Conference on High Energy Physics, 2000, Osaka, Japan, ALEPH 2000-062.

[3] CLEO Collaboration, M. Chadha et al., Phys. Rev. D 58, 032002 (1998).

[4] S. Hashimoto, Nucl. Phys. B (Proc. Suppl.) 83-84, 3 (2000).

[5] T. Draper, Nucl. Phys. B (Proc. Suppl.) 73, 43 (1999).

[6] CP-PACS Collaboration, S. Aoki et al., Phys. Rev. Lett. 84, 238 (2000).

[7] M.J. Booth, Phys. Rev. D 51, 2338 (1995).

[8] S.R. Sharpe and Y. Zhang, Phys. Rev. D 53, 5125 (1996).
[9] C. Bernard et al., Phys. Rev. Lett. 81, 4812 (1998); Nucl. Phys. B (Proc. Suppl.) 83-84, 289 (2000).

[10] S. Collins, C.T. Davies, U.M. Heller, A. Ali Khan, J. Shigemitsu, J. Sloan, and C. Morningstar, Phys. Rev. D 60, 074504 (1999).

[11] B. Sheikholeslami and R. Wohlert, Nucl. Phys. B259, 572 (1985).

[12] A.X. El-Khadra, A.S. Kronfeld, and P.B. Mackenzie, Phys. Rev. D 55, 3933 (1997).

[13] Y. Iwasaki, Nucl. Phys. B258, 141 (1985); University of Tsukuba Report No. UTHEP-118, 1983.

[14] CP-PACS Collaboration, A. Ali Khan et al. (in preparation).

[15] S. Aoki et al., Nucl. Phys. B540, 501 (1999). 
[16] CP-PACS Collaboration, S. Aoki et al., Phys. Rev. D 60, 114508 (1999).

[17] E. Eichten and B. Hill, Phys. Lett. B 234, 511 (1990); H. Georgi, ibid. 240, 447 (1990).

[18] E. Eichten, Nucl. Phys. B (Proc. Suppl.) 4, 170 (1988).

[19] B.A. Thacker and G.P. Lepage, Phys. Rev. D 43, 196 (1991).

[20] G.P. Lepage et al., Phys. Rev. D 46, 4052 (1992).

[21] B.P.G. Mertens, A.S. Kronfeld, and A.X. El-Khadra, Phys. Rev. D 58, 034505 (1998).

[22] Y. Kuramashi, Phys. Rev. D 58, 034507 (1998).

[23] S. Aoki, S. Hashimoto, K.-I. Ishikawa, and T. Onogi (unpublished).

[24] G.P. Lepage and P.B. Mackenzie, Phys. Rev. D 48, 2250 (1993).

[25] C. W. Bernard, J. N. Labrenz, and A. Soni, Phys. Rev. D 49, 2536 (1994).
[26] JLQCD Collaboration, S. Aoki et al., Phys. Rev. Lett. 80, 5711 (1998).

[27] A.X. El-Khadra, A.S. Kronfeld, P.B. Mackenzie, S.M. Ryan, and J.N. Simone, Phys. Rev. D 58, 014506 (1998).

[28] R. Burkhalter, Nucl. Phys. B (Proc. Suppl.) 73, 3 (1999).

[29] CP-PACS Collaboration, A. Ali Khan et al., Phys. Rev. Lett. 85, 4674 (2000).

[30] UKQCD Collaboration, H.P. Shanahan et al., Phys. Rev. D 55, 1548 (1997).

[31] S. Aoki, K. Nagai, Y. Taniguchi, and A. Ukawa, Phys. Rev. D 58, 074505 (1998).

[32] K.-I. Ishikawa, T. Onogi, and N. Yamada, Nucl. Phys. B (Proc. Suppl.) 83, 301 (2000); K.-I. Ishikawa (private communication).

[33] L. Wolfenstein, Phys. Rev. Lett. 51, 1945 (1983). 